\title{
Title of Manuscript: Glycosides from the Stem Bark of Fraxinus sieboldiana
}

Author list: Sheng Lin, Sujuan Wang, Mingtao Liu, Maoluo Gan, Shuai Li, Yongchun Yang, Yinghong Wang, Wenyi He, Jiangong Shi*

\section{Supporting Information Available}

\footnotetext{
* To whom correspondence should be addressed. Tel: 86-10-83154789. Fax: 86-10-63017757. E-mail: shijg@imm.ac.cn
} 


\section{The List of Contents}

\begin{tabular}{|c|c|c|}
\hline no. & Content & Page \\
\hline 1 & The ${ }^{1} \mathrm{H}$ NMR Spectrum of Fraxinuacidoside (1) in $\mathrm{MeOH}-d_{4}$ & S3 \\
\hline 2 & The ${ }^{13} \mathrm{C}$ NMR Spectrum of Fraxinuacidoside (1) in $\mathrm{MeOH}-d_{4}$ & S4 \\
\hline 3 & The DEPT Spectrum of Fraxinuacidoside (1) in $\mathrm{MeOH}-d_{4}$ & S5 \\
\hline 4 & The NOE Difference Spectrum 1 of Fraxinuacidoside (1) in MeOH- $d_{4}$ & S6 \\
\hline 5 & The NOE Difference Spectrum 2 of Fraxinuacidoside (1) in MeOH- $d_{4}$ & S7 \\
\hline 6 & The ${ }^{1} \mathrm{H}$ NMR Spectrum of compound 1a in $\mathrm{MeOH}-d 4$ & S8 \\
\hline 7 & The ${ }^{13} \mathrm{C}$ NMR Spectrum of compound 1a in MeOH- $d 4$ & S9 \\
\hline 8 & The ${ }^{1}$ H NMR Spectrum of compound 2 in DMSO- $d_{6}$ & S10 \\
\hline 9 & The ${ }^{13} \mathrm{C}$ NMR Spectrum of compound $\mathbf{2}$ in DMSO- $d_{6}$ & S11 \\
\hline 10 & The DEPT Spectrum of compound 2 in DMSO- $d_{6}$ & S12 \\
\hline 11 & The NOE Difference Spectrum 1 of compound 2 in DMSO- $d_{6}+\mathrm{D}_{2} \mathrm{O}$ & S13 \\
\hline 12 & The NOE Difference Spectrum 2 of compound 2 in DMSO- $d_{6}+\mathrm{D}_{2} \mathrm{O}$ & S14 \\
\hline 13 & The ${ }^{1} \mathrm{H}$ NMR Spectrum of compound 3 in DMSO- $d_{6}$ & S15 \\
\hline 14 & The ${ }^{13} \mathrm{C}$ NMR Spectrum of compound $\mathbf{3}$ in DMSO- $d_{6}$ & S16 \\
\hline 15 & The NOE Difference Spectrum of compound 3 in DMSO- $d_{6}$ & S17 \\
\hline 16 & The ${ }^{1}$ H NMR Spectrum of compound $\mathbf{4}$ in DMSO- $d_{6}$ & S18 \\
\hline 17 & The ${ }^{13} \mathrm{C}$ NMR Spectrum of compound $\mathbf{4}$ in DMSO- $d_{6}$ & S19 \\
\hline 18 & The ${ }^{1} \mathrm{H}$ NMR Spectrum of compound 5 in DMSO- $d_{6}$ & S20 \\
\hline 19 & The ${ }^{13} \mathrm{C}$ NMR Spectrum of compound 5 in DMSO- $d_{6}$ & S21 \\
\hline 20 & The DEPT Spectrum of compound 5 in DMSO- $d_{6}$ & S22 \\
\hline 21 & The ${ }^{1} \mathrm{H}$ NMR Spectrum of compound 6 in DMSO- $d_{6}$ & S23 \\
\hline 22 & The ${ }^{13} \mathrm{C}$ NMR Spectrum of compound 6 in DMSO- $d_{6}$ & S24 \\
\hline 23 & The ${ }^{1} \mathrm{H}$ NMR Spectrum of compound 7 in $\mathrm{D}_{2} \mathrm{O}$ & S25 \\
\hline 24 & The ${ }^{13} \mathrm{C}$ NMR Spectrum of compound 7 in $\mathrm{D}_{2} \mathrm{O}$ & S26 \\
\hline 25 & The DEPT Spectrum of compound 7 in $\mathrm{D}_{2} \mathrm{O}$ & S27 \\
\hline 26 & The ${ }^{1} \mathrm{H}$ NMR Spectrum of compound 8 in $\mathrm{D}_{2} \mathrm{O}$ & S28 \\
\hline 27 & The ${ }^{13} \mathrm{C}$ NMR Spectrum of compound 8 in $\mathrm{D}_{2} \mathrm{O}$ & S29 \\
\hline 28 & The DEPT Spectrum of compound 8 in $\mathrm{D}_{2} \mathrm{O}$ & S30 \\
\hline 29 & The ${ }^{1} \mathrm{H}$ NMR Spectrum of compound 9 in $\mathrm{D}_{2} \mathrm{O}$ & S31 \\
\hline 30 & The ${ }^{13} \mathrm{C}$ NMR Spectrum of compound 9 in $\mathrm{D}_{2} \mathrm{O}$ & S32 \\
\hline 31 & The DEPT Spectrum of compound $\mathbf{9}$ in $\mathrm{D}_{2} \mathrm{O}$ & S33 \\
\hline 32 & The HPLC Spectrum of compounds $\mathbf{8}$ and $\mathbf{9}$ & S34 \\
\hline 33 & SI Table $1 .{ }^{13} \mathrm{C}$ NMR Data of 7-9 in Different Solvents (DMSO- $d_{6}, \mathrm{C}_{5} \mathrm{D}_{5} \mathrm{~N}, \mathrm{D}_{2} \mathrm{O}$ ) & S35 \\
\hline 34 & $\begin{array}{l}\text { SI Table } 2 .{ }^{13} \mathrm{C} \text { NMR Data of 7a-9a in Different Solvents (DMSO- } d_{6}, \mathrm{C}_{5} \mathrm{D}_{5} \mathrm{~N}, \mathrm{MeOH}-d_{4} \text {, } \\
\text { and } \mathrm{Me}_{2} \mathrm{CO}-d_{6} \text { ) }\end{array}$ & S36 \\
\hline 35 & SI Table 3. ${ }^{1} \mathrm{H}$ NMR Data of 7-9 in DMSO- $d_{6}$ and $\mathrm{C}_{5} \mathrm{D}_{5} \mathrm{~N}$ & S37 \\
\hline 36 & SI Table 4. ${ }^{1} \mathrm{H}$ NMR Data of 7a-9a in $\mathrm{Me}_{2} \mathrm{CO}-d_{6}, \mathrm{MeOH}-d_{4}$, DMSO- $d_{6}$, and $\mathrm{C}_{5} \mathrm{D}_{5} \mathrm{~N}$ & S38 \\
\hline
\end{tabular}




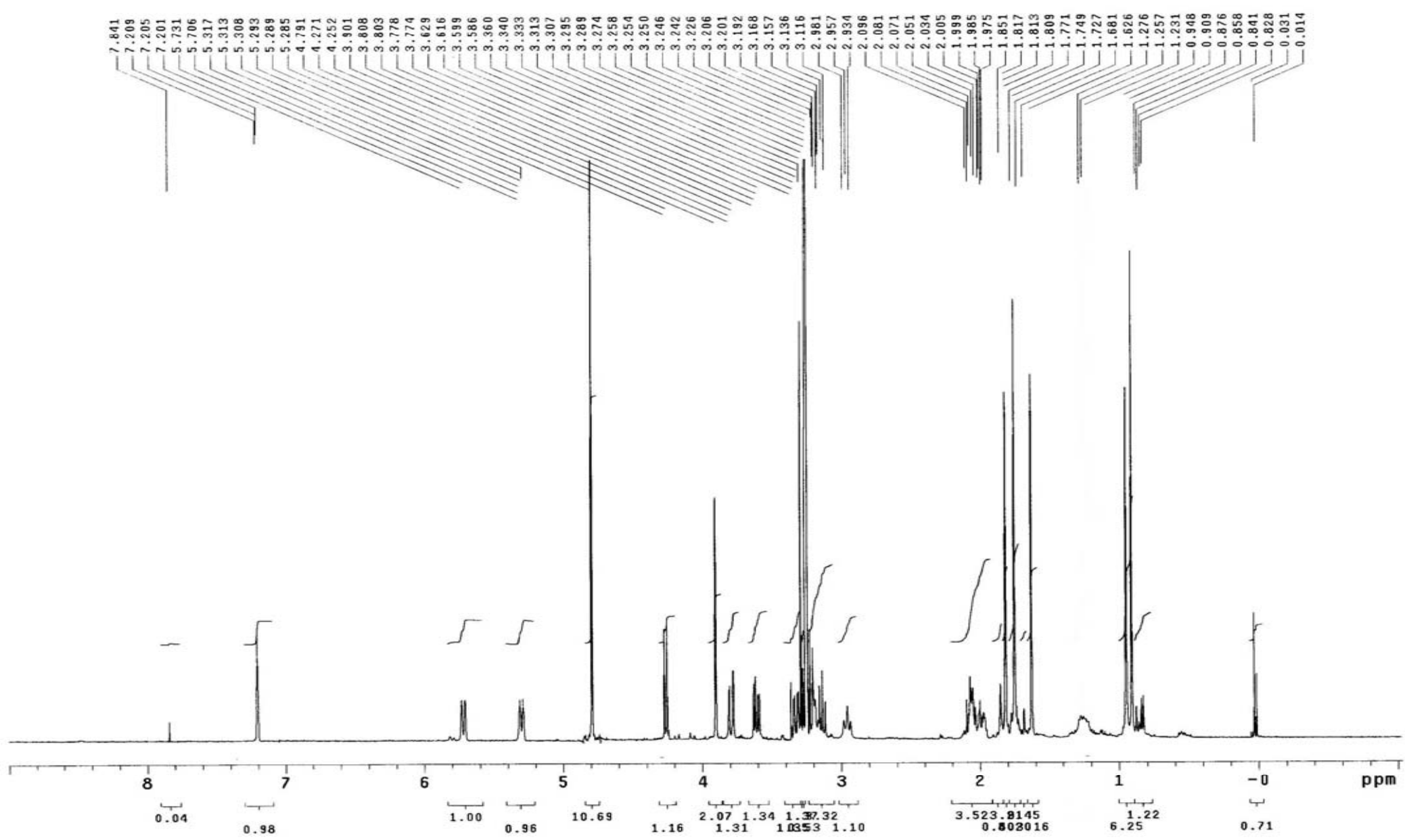

The ${ }^{1} \mathrm{H}$ NMR Spectrum of Fraxinuacidoside (1) in MeOH- $d_{4}$ 

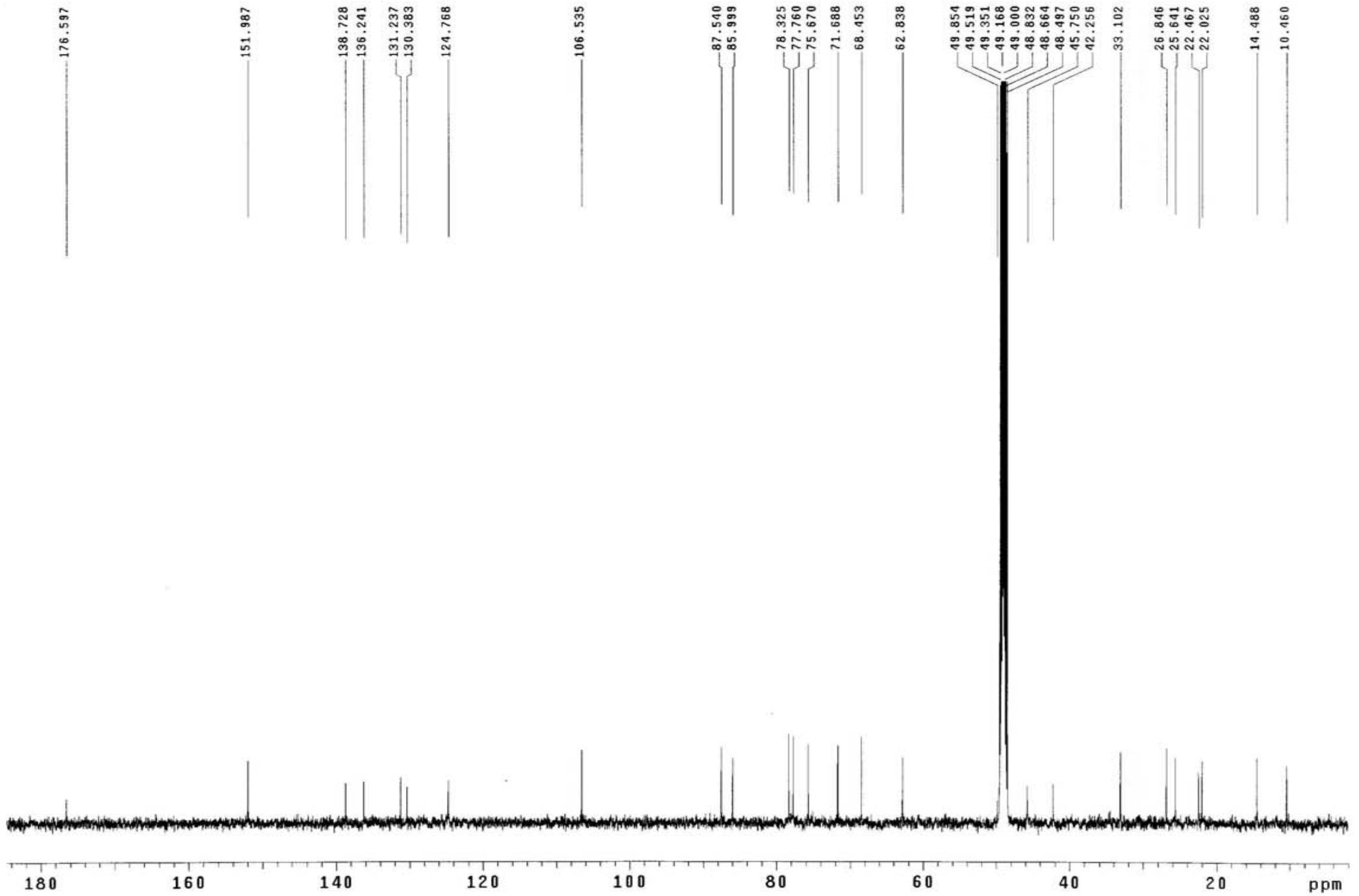

The ${ }^{13} \mathrm{C}$ NMR Spectrum of Fraxinuacidoside (1) in MeOH-d $\mathrm{d}_{4}$ 
烈
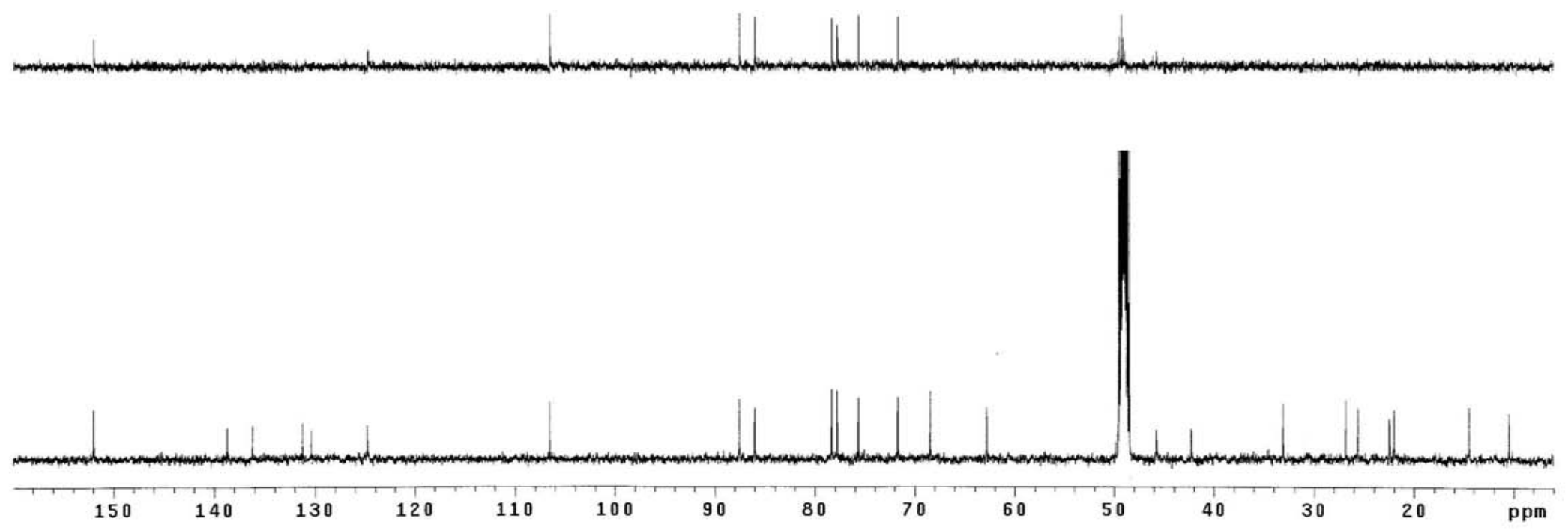

The DEPT Spectrum of Fraxinuacidoside (1) in MeOH- $d_{4}$ 

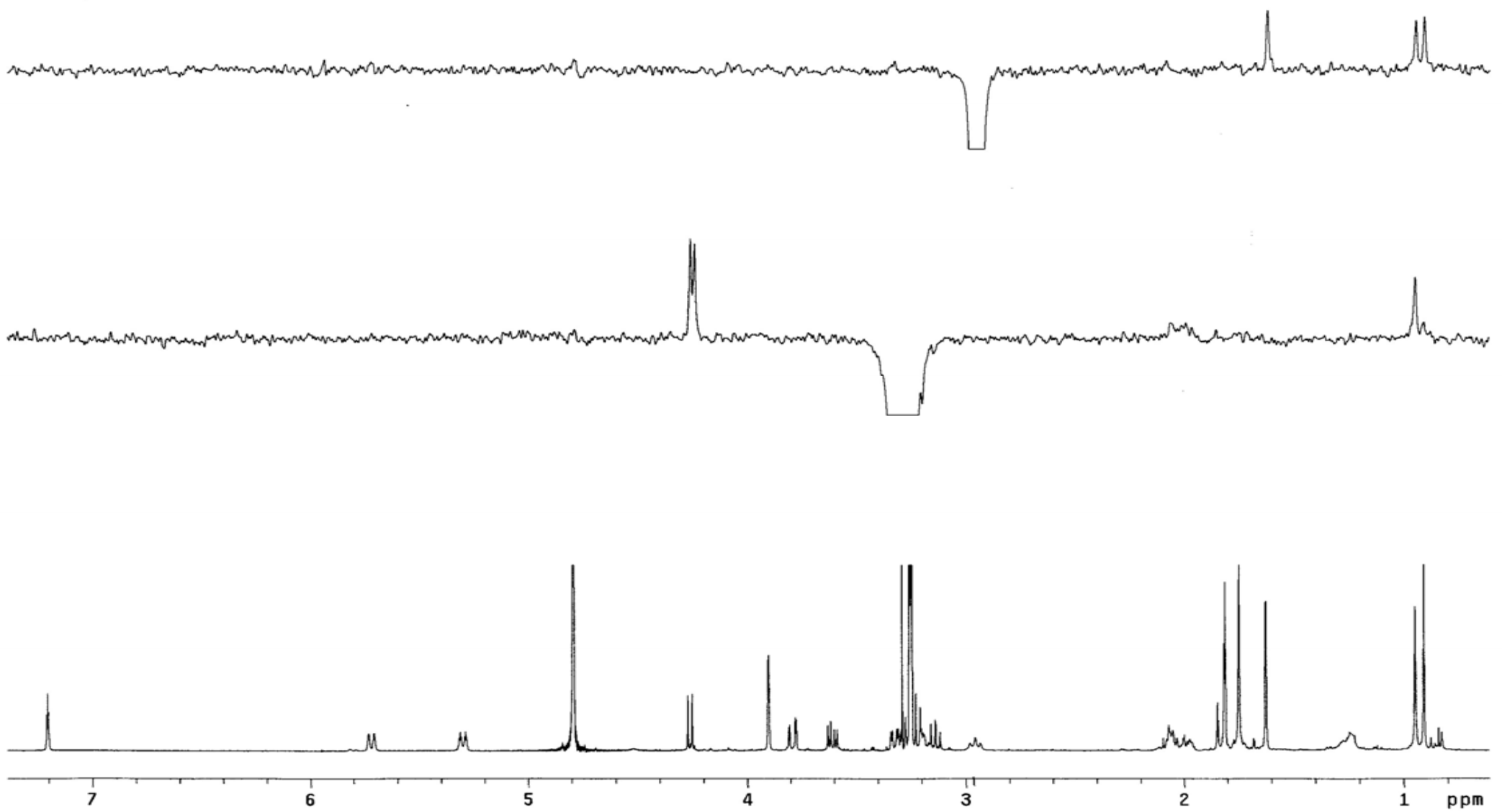

The NOE Difference Spectrum 1 of Fraxinuacidoside (1) in MeOH-d $d_{4}$ 


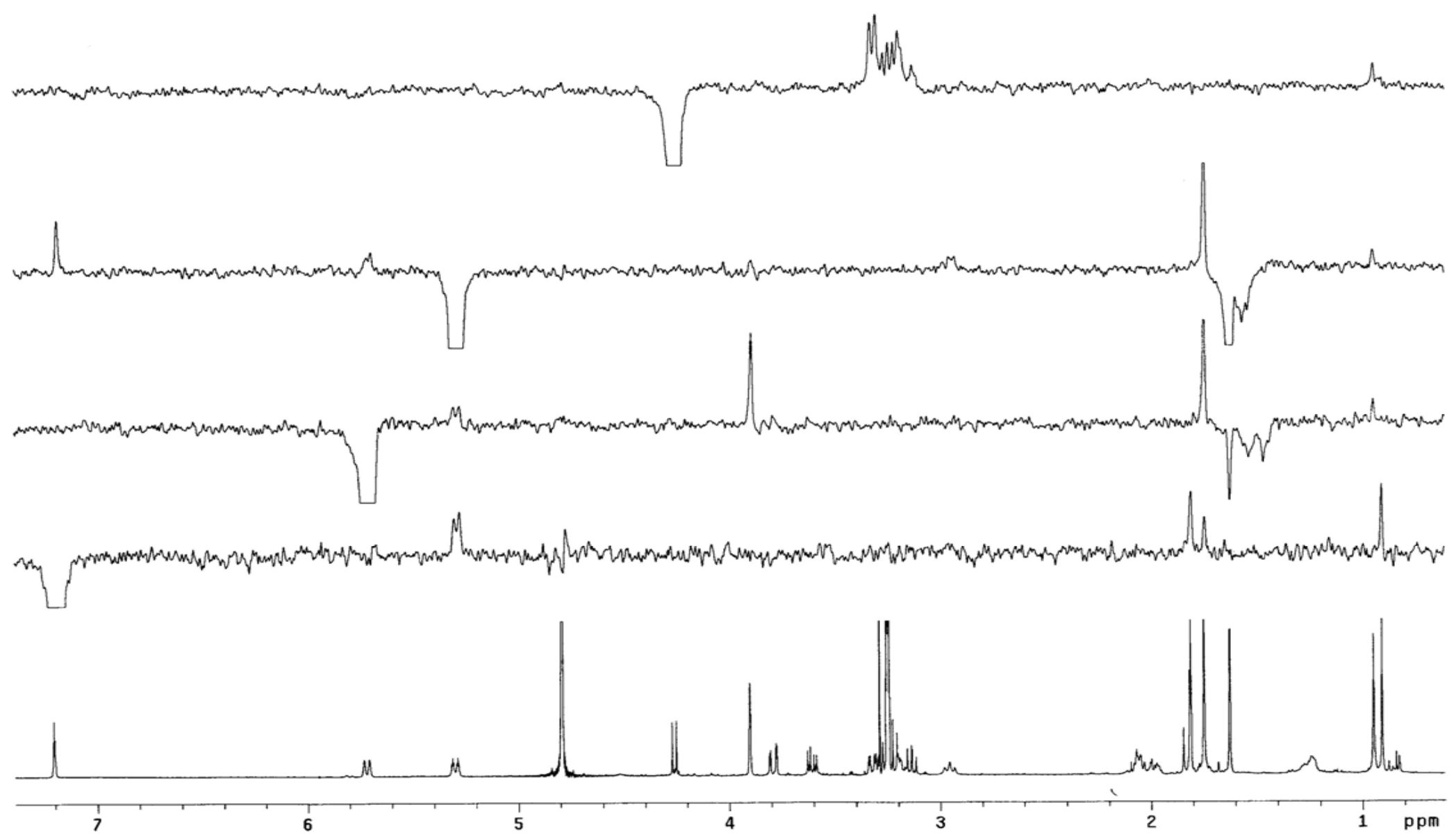

The NOE Difference Spectrum 2 of Fraxinuacidoside (1) in MeOH-d $\mathrm{d}_{4}$ 


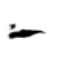

MERCURY-400 1H-NMR FMAR-M-44A IN CD30D 2006.11.30

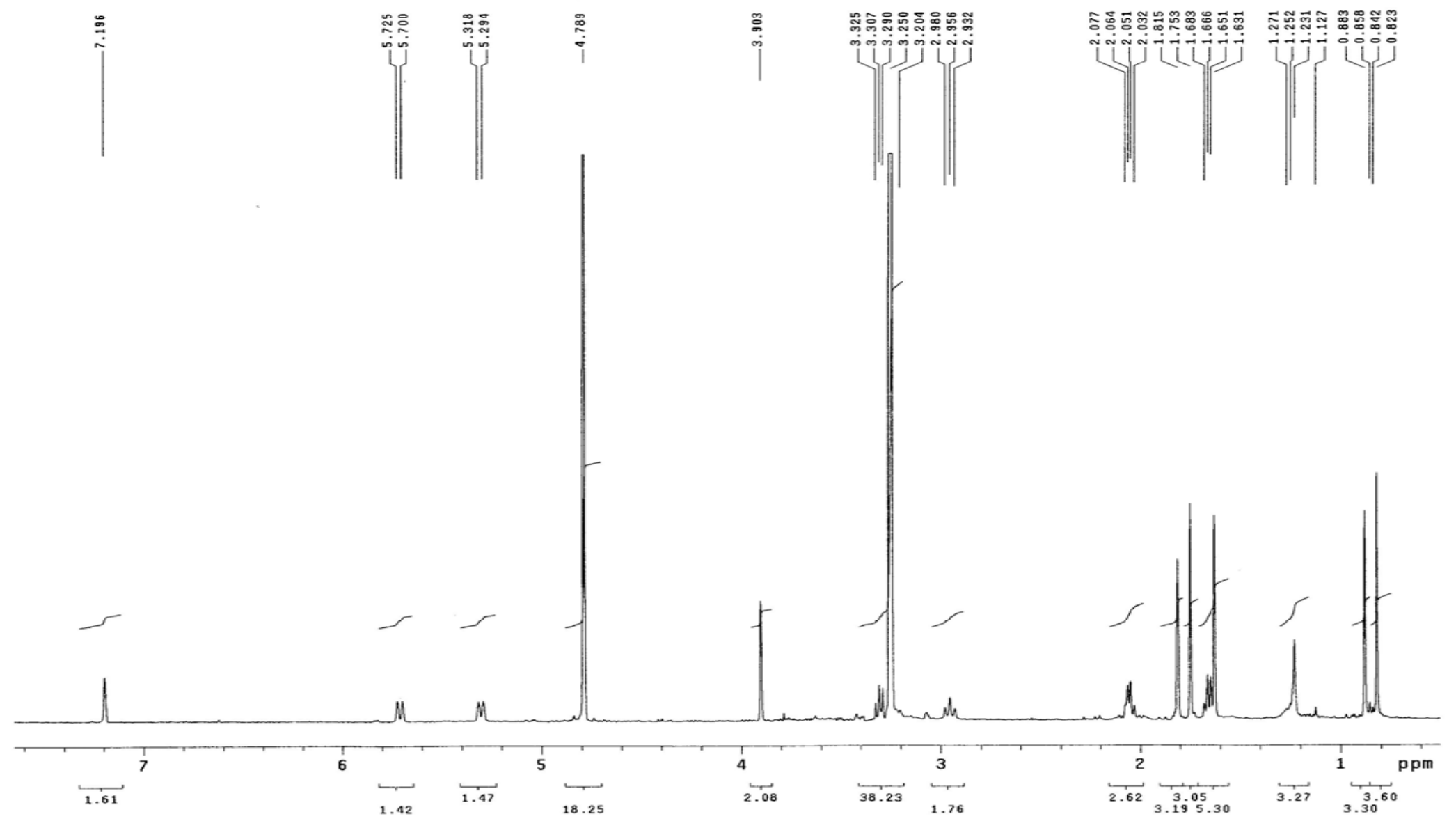

The ${ }^{1} \mathrm{H}$ NMR Spectrum of Compound (1a) in MeOH- $d_{4}$ 
INOVA-500 13C-NMR FMAR-M-44A IN CD30D 2006.12.03
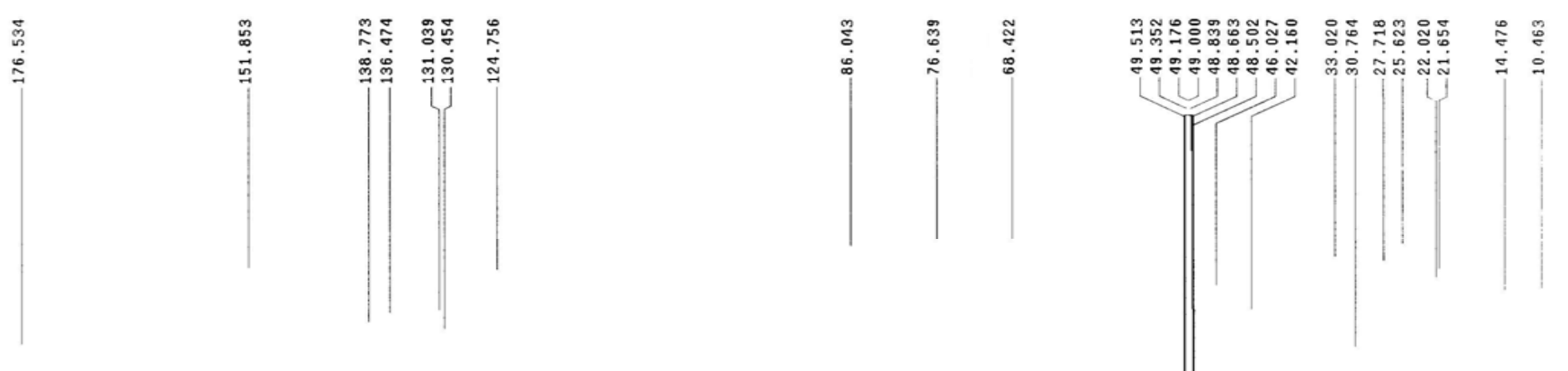

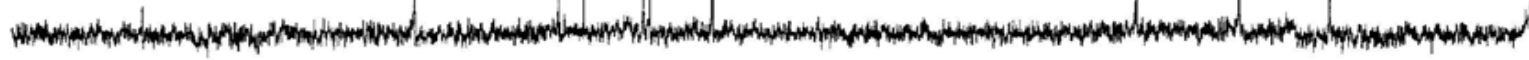

The ${ }^{13} \mathrm{C}$ NMR Spectrum of Compound (1a) in MeOH- $d_{4}$ 
$--\infty$

INOVA-500 1H-NMR FMAR-M-33 IN DMSO 2005.12.2

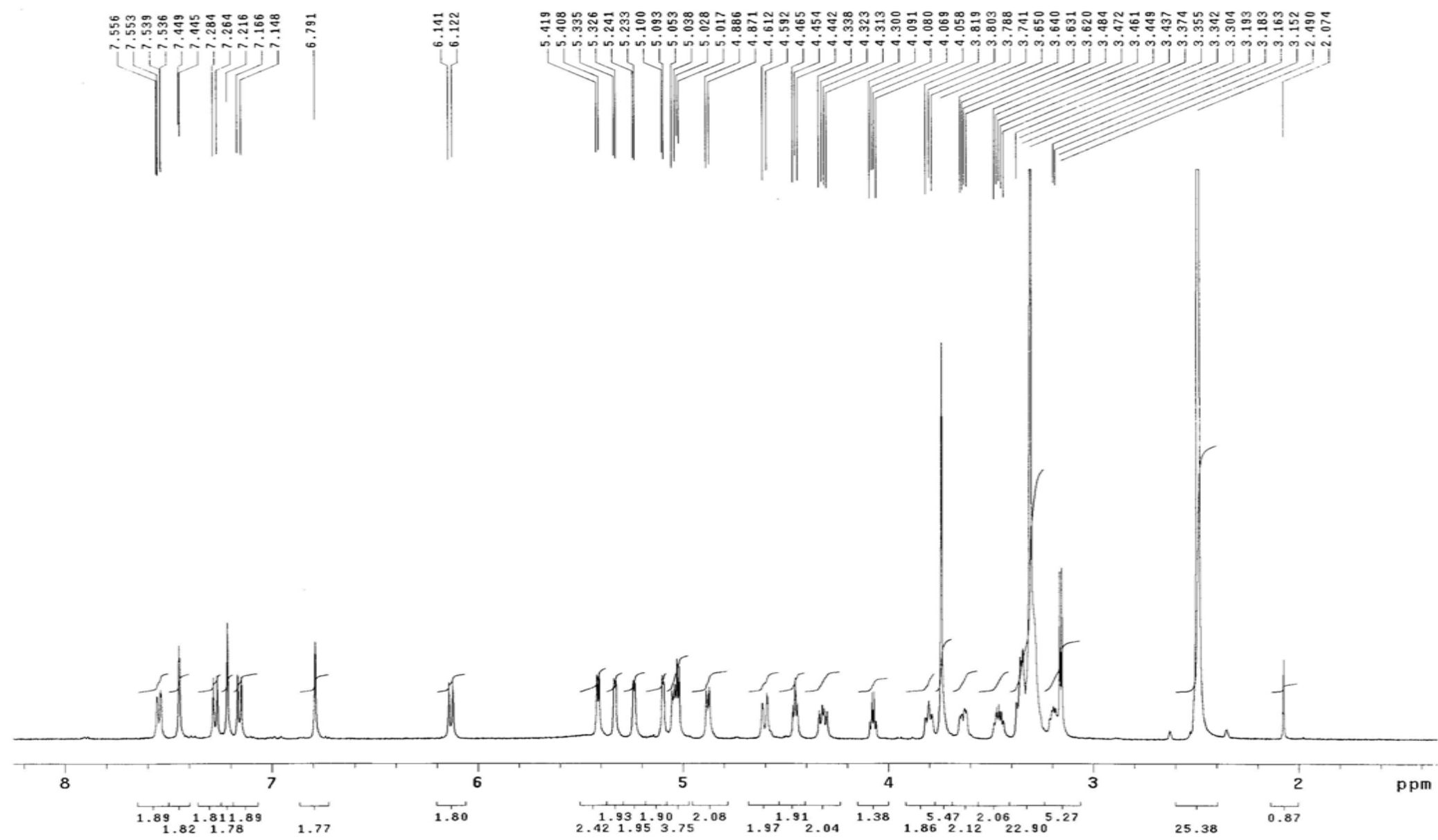

The ${ }^{1} \mathrm{H}$ NMR Spectrum of Compound 2 in DMSO- $d_{6}$ 
-16 INOVA-500 13C-NMR F-MAR-M-33 IN DMSO
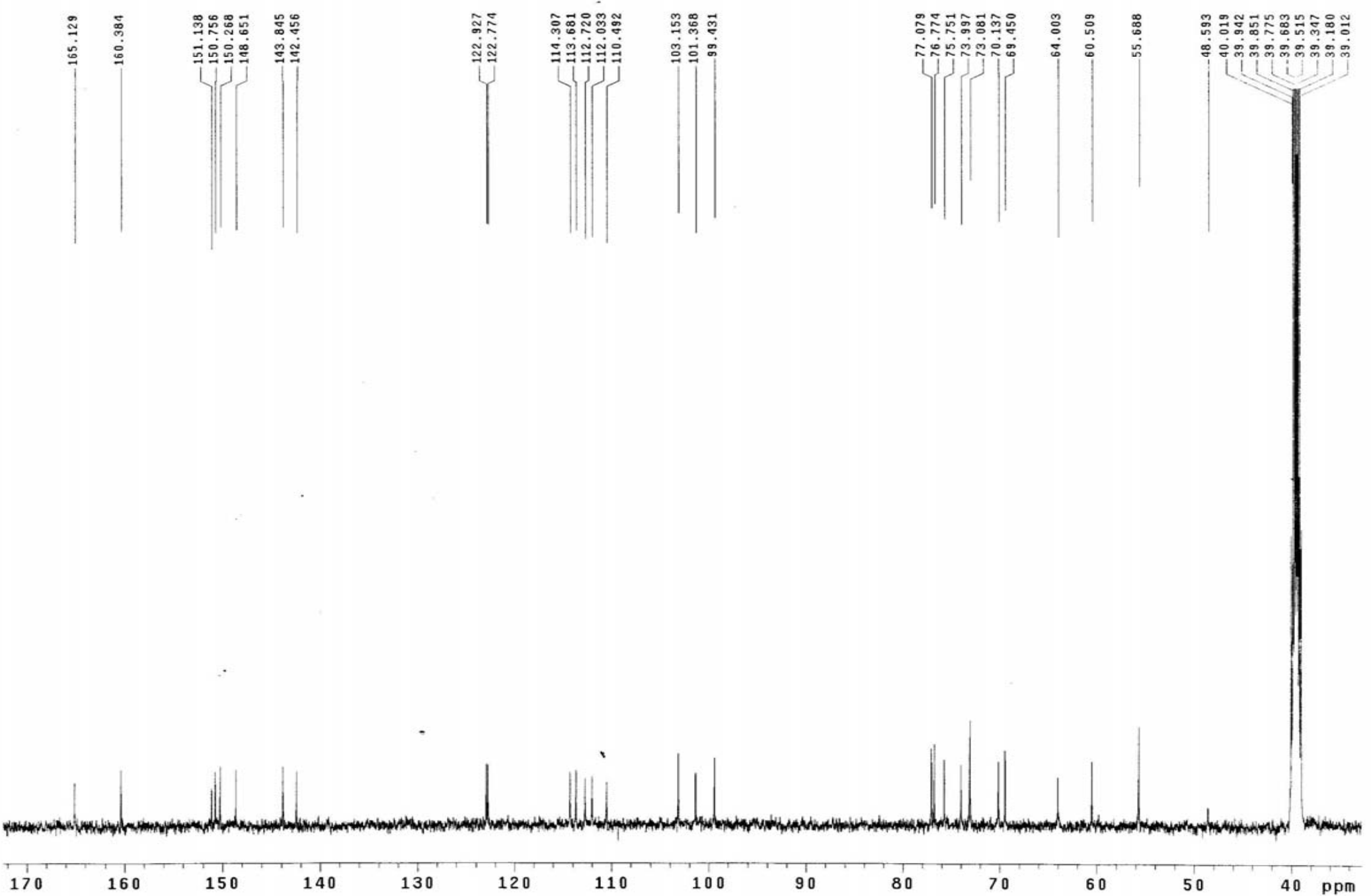

The ${ }^{13}$ C NMR Spectrum of Compound 2 in DMSO- $d_{6}$ 

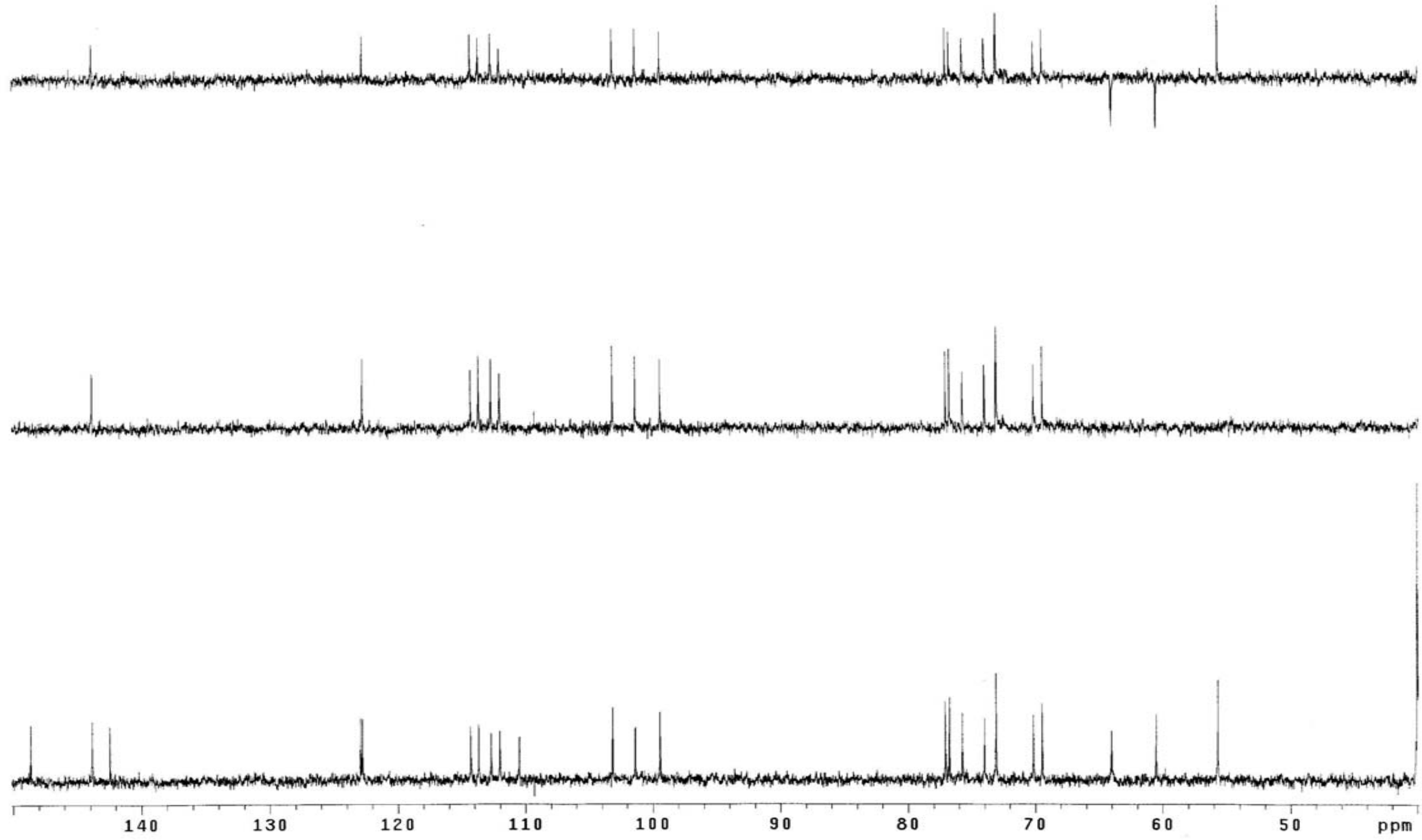

The DEPT Spectrum of Compound 2 in DMSO- $d_{6}$ 

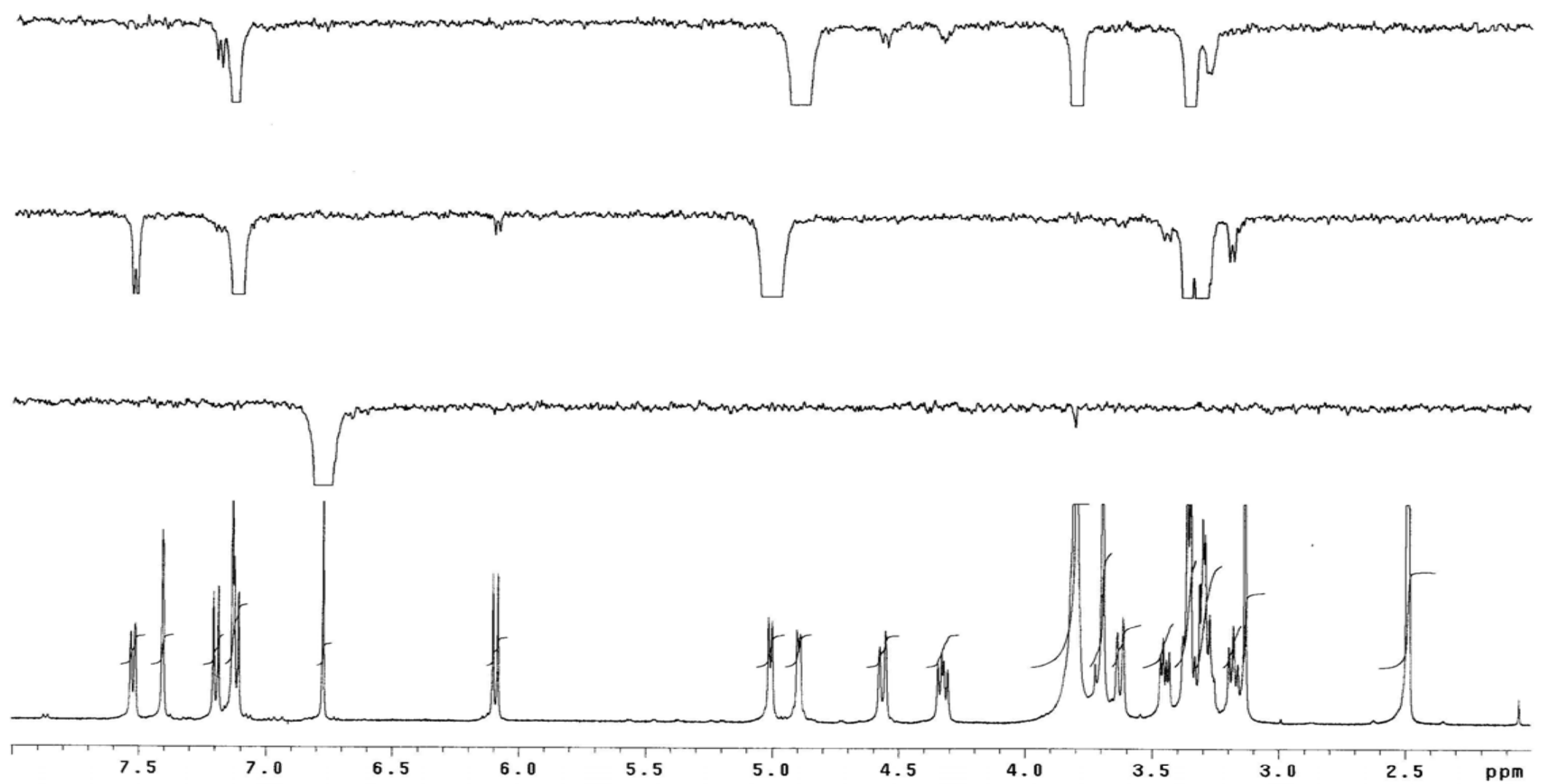

The NOE Difference Spectrum 1 of Compound 2 in DMSO- $d_{6}+D_{2} \mathrm{O}$ 
INOVA-501 NOESY10 F.MAR-M-33 IN DMSO+D20 06.04.10 Archive directory: /export/home/vnmr1/vnmrsys/data
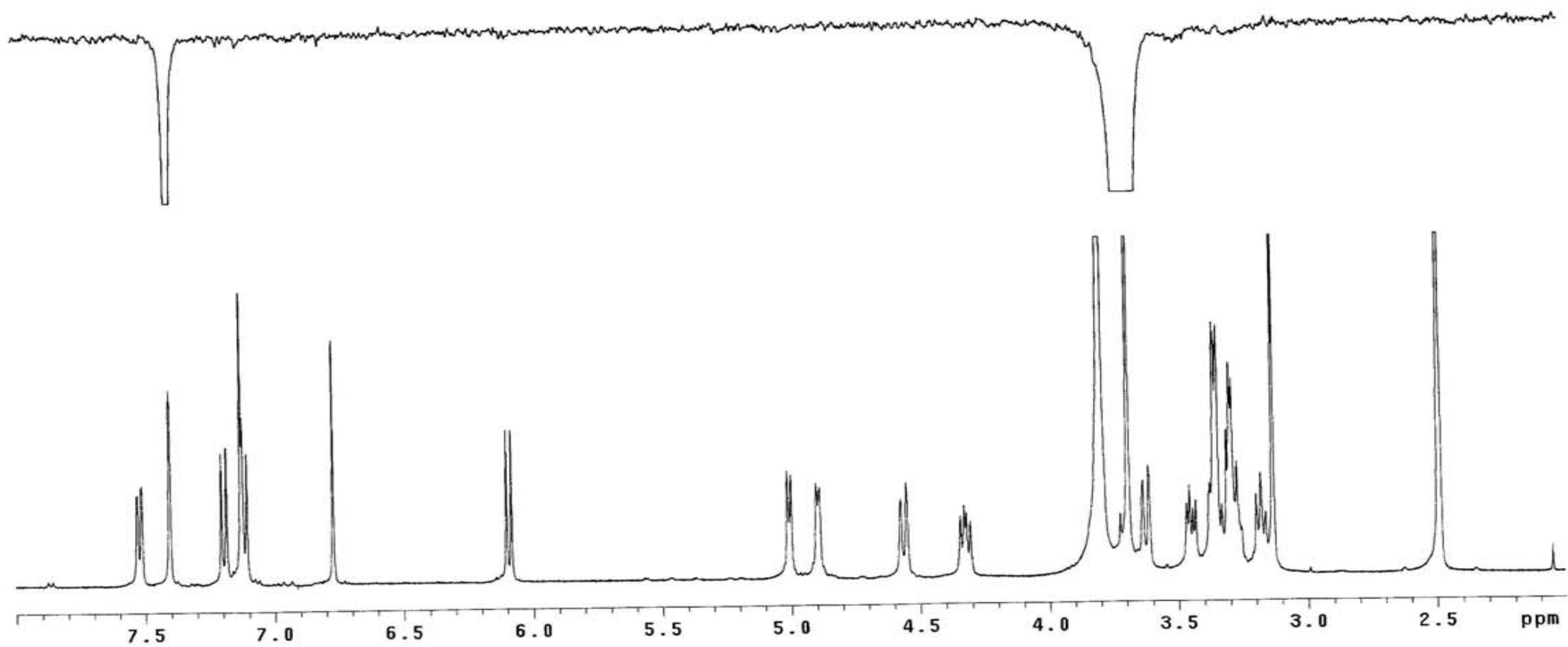

The NOE Difference Spectrum 2 of Compound 2 in DMSO- $d_{6}+D_{2} \mathrm{O}$ 
INOVA-501 1H-NMR F.MAR-M-8 IN DMSO 05.08.24

Archive directory: /export/home/vnmr1/vnmrsys/data

Pulse Sequence: s2pur

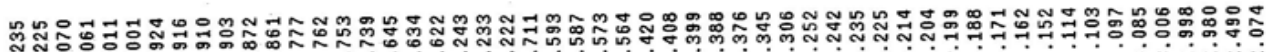

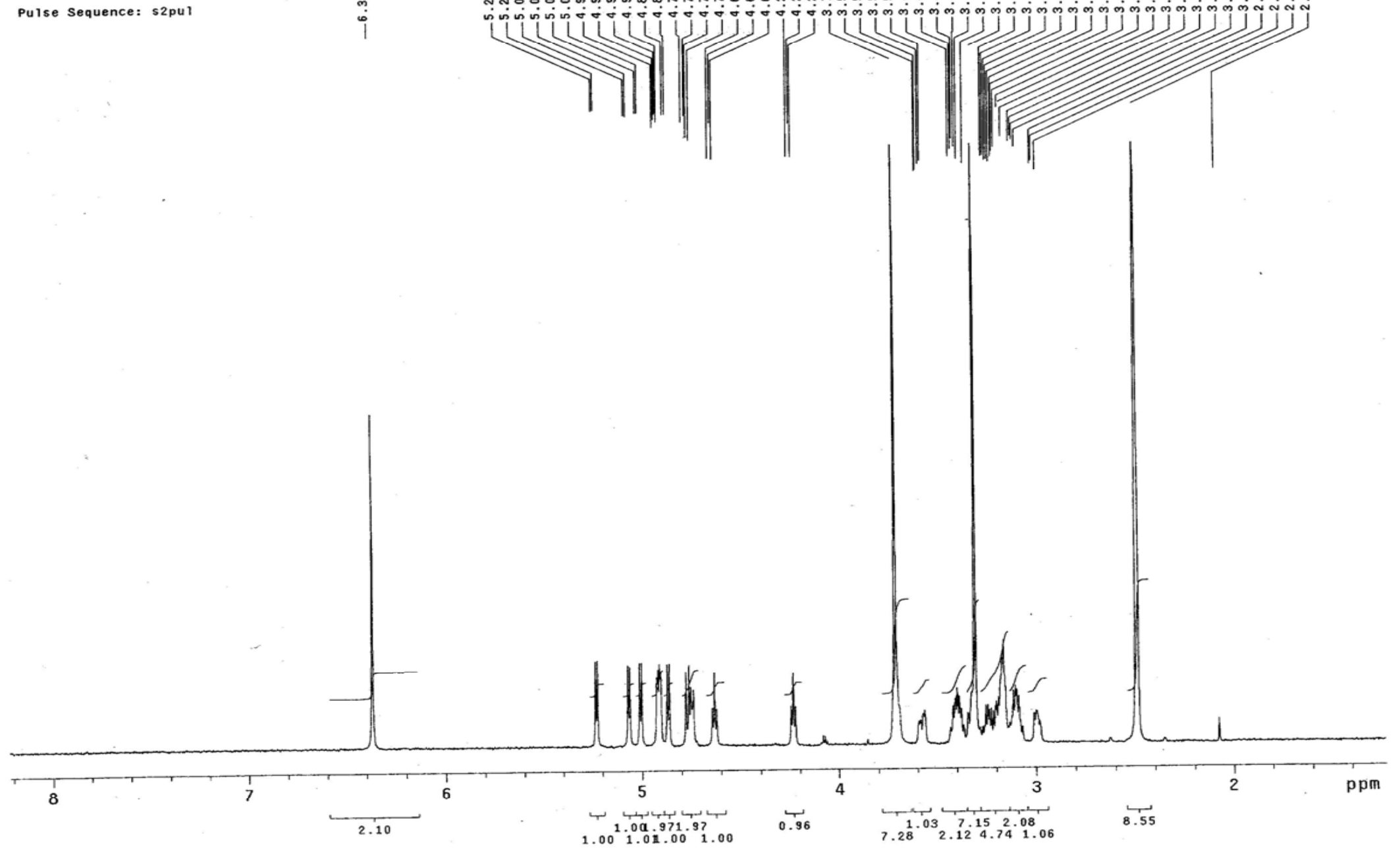

The ${ }^{1} \mathrm{H}$ NMR Spectrum of Compound 3 in DMSO-d 6 
INOVA-500 13C-NMR F-MAR-M-8 IN OMSO 2005.08.25
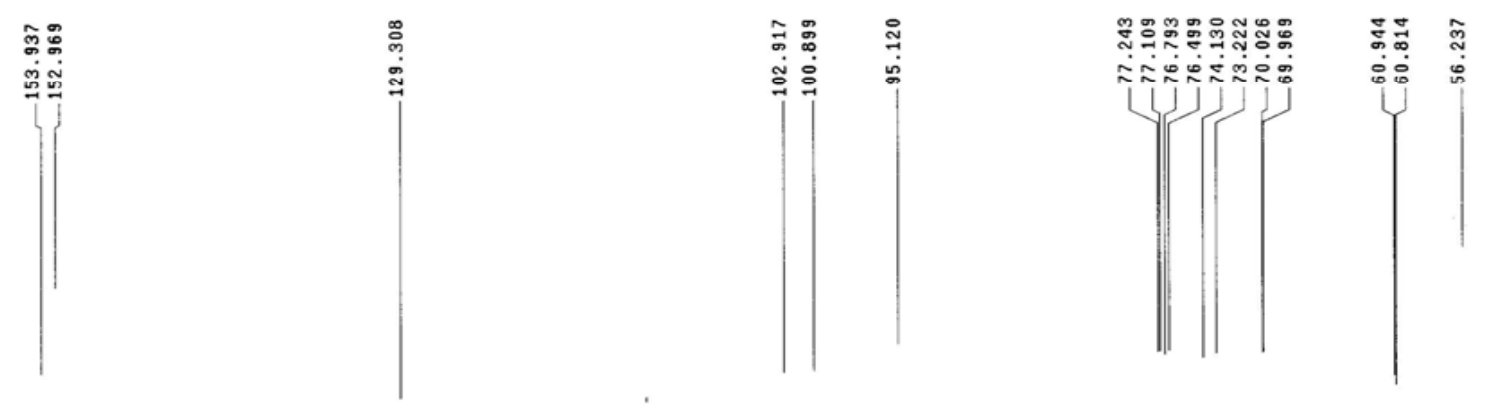

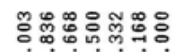

广क्ल心
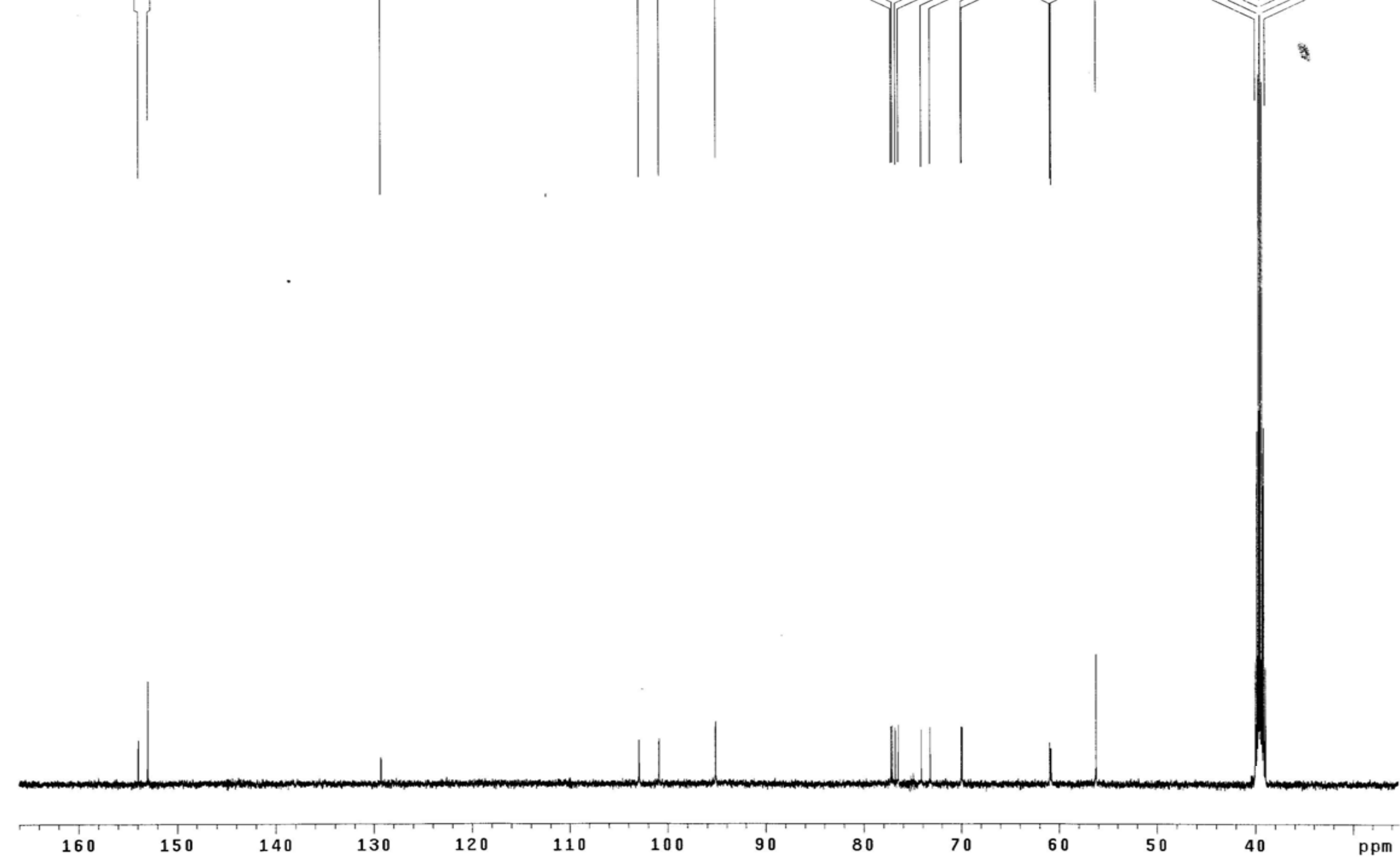

The ${ }^{13} \mathrm{C}$ NMR Spectrum of Compound 3 in DMSO- $d_{6}$ 
INOVA-501 NOESY10 F.MAR-M-8 IN OKSO 05.08.31

Archive directory: /export/home/vnmr1/vnmrsys/data

Sample directory:
File: PROTON

PUlse Sequence: NOESY10
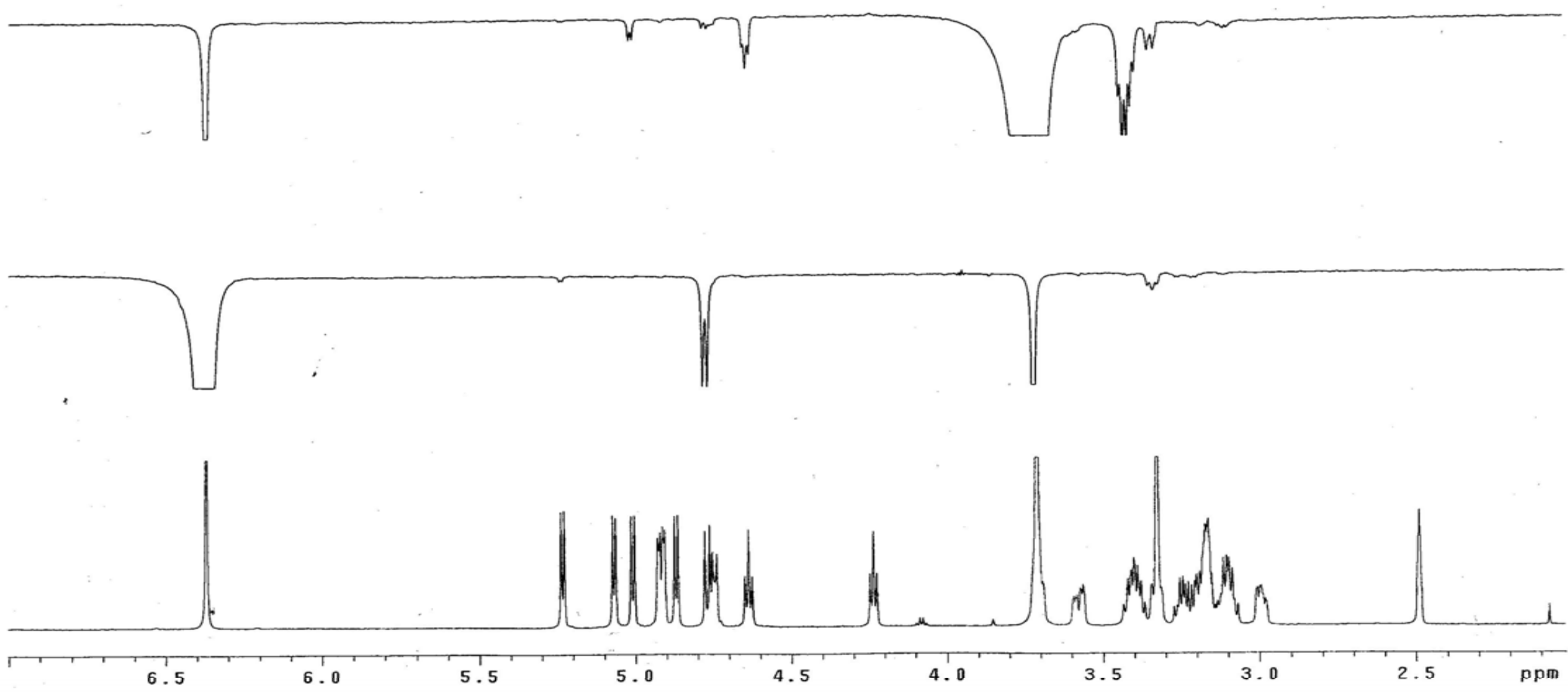

The NOE Difference Spectrum of Compound 3 in DMSO- $d_{6}$ 
INOVA-501 1H-NMR F.mar-M24 IN DMSO 05.10.25

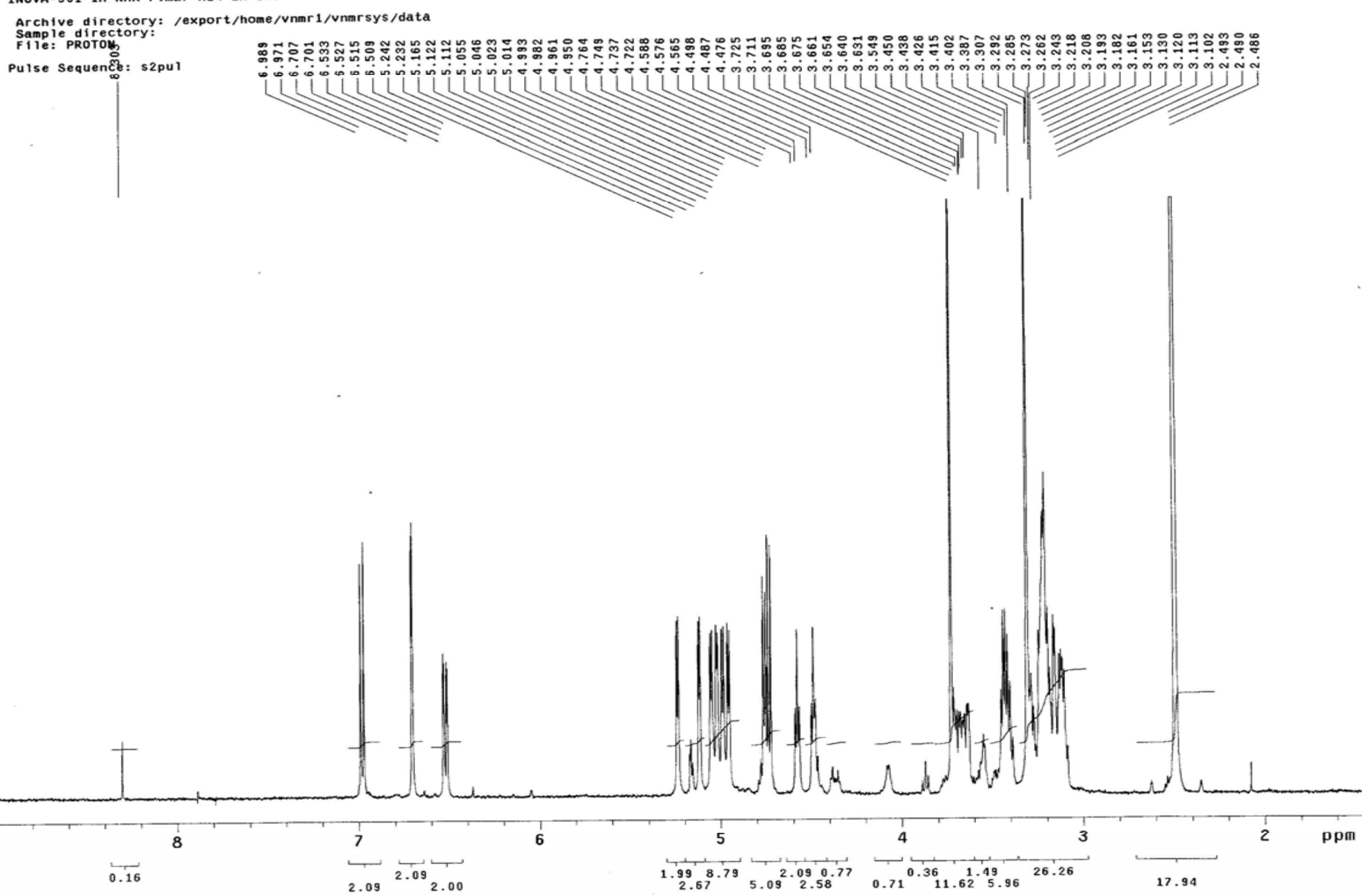

The ${ }^{1} \mathrm{H}$ NMR Spectrum of Compound 4 in DMSO- $d_{6}$ 
INOVA-500 13C-NMR FMAR-M-24 IN DMSO
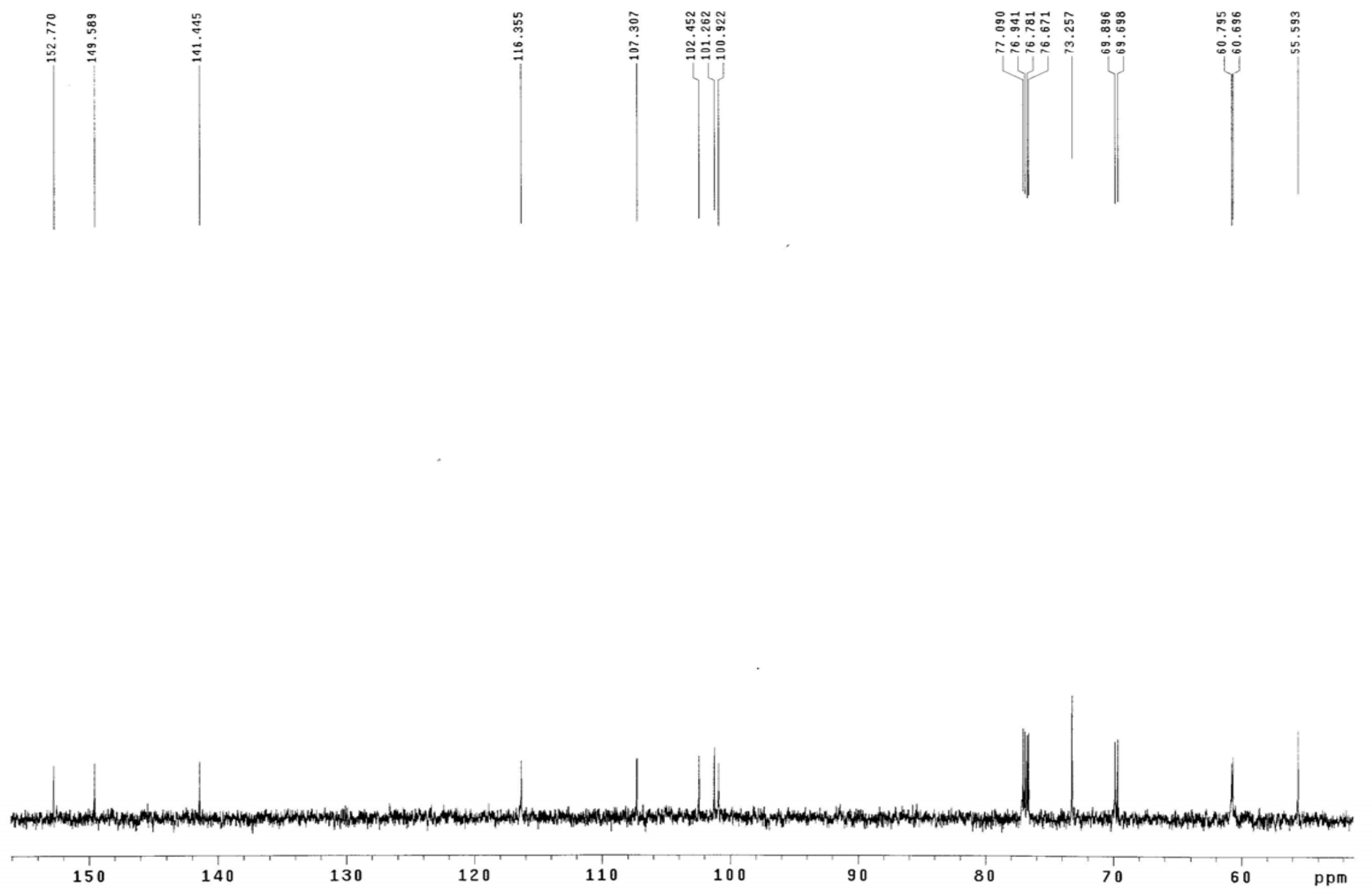

The ${ }^{13} \mathrm{C}$ NMR Spectrum of Compound 4 in DMSO- $d_{6}$ 
INOYA-501 1H-NMR F.MAR-M-6 IN OMSO 05.08.30 Archive directory: /export/home/vnmr 1/vnmrsys/data

Sile: PROTON

Pulse Sequence: s2pur

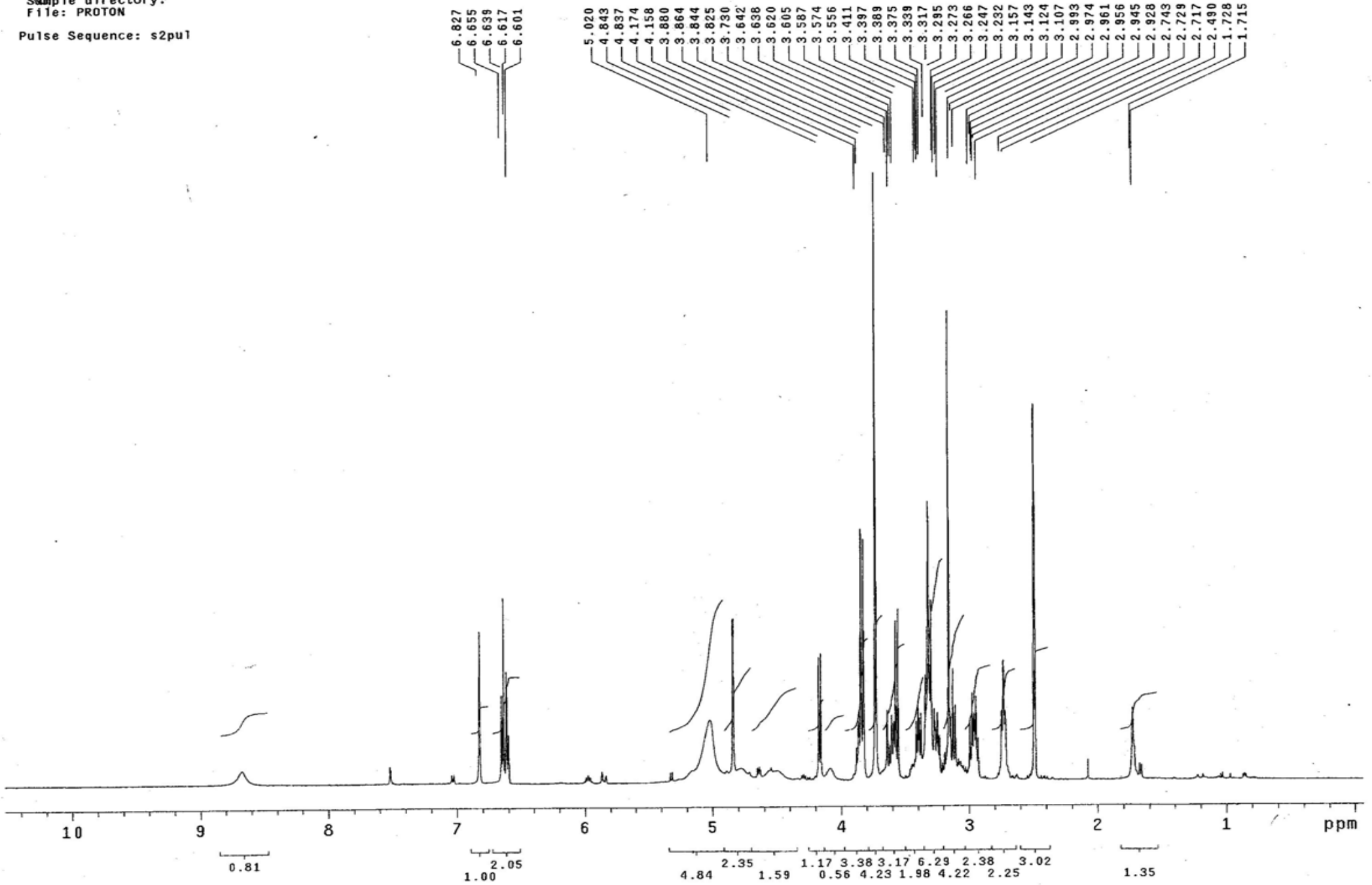

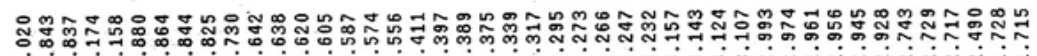

The ${ }^{1} \mathrm{H}$ NMR Spectrum of Compound 5 in DMSO- $d_{6}$ 
INOVA-500 13C-NMR F-MAR-M-6 IN DMSO
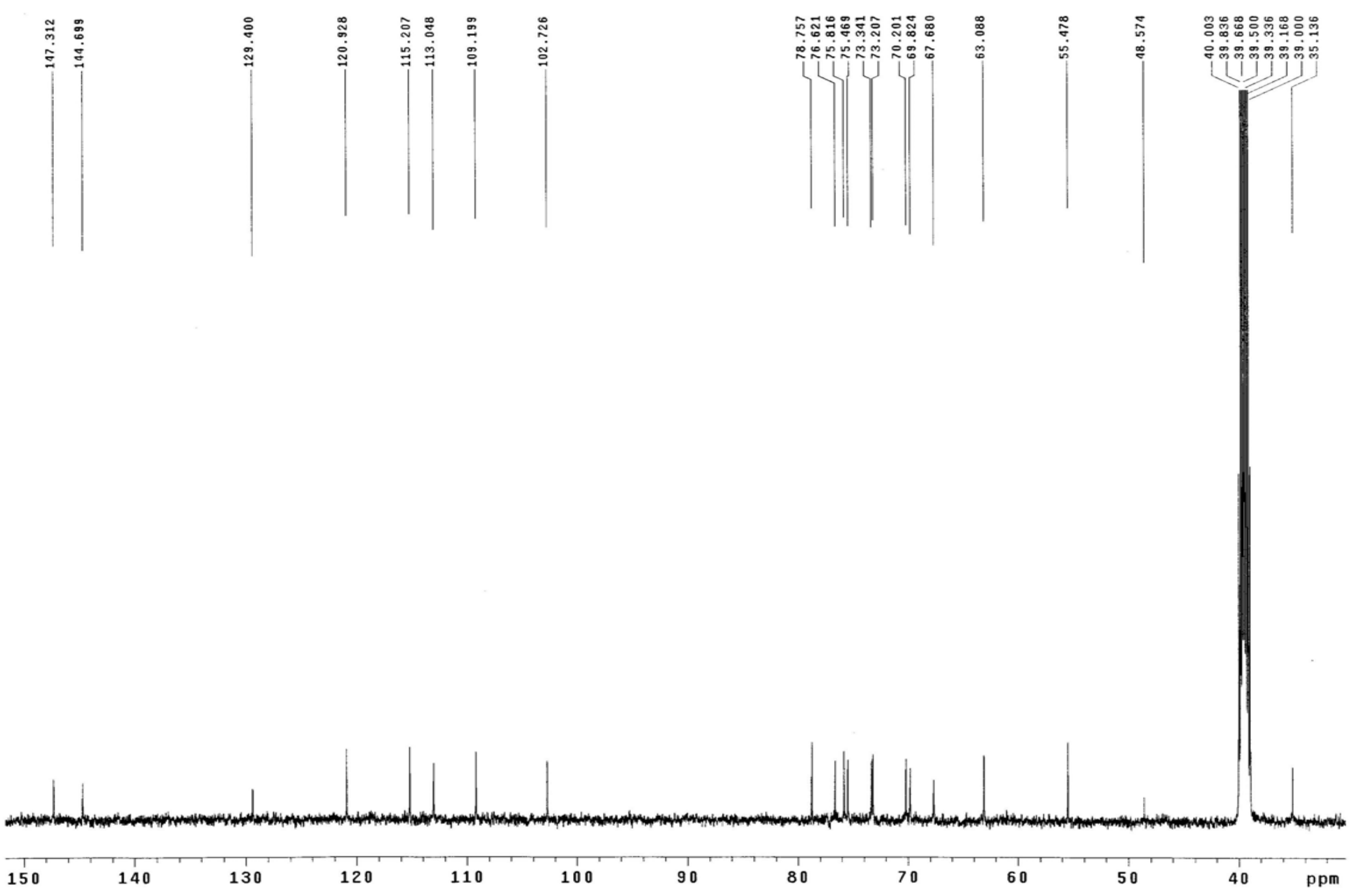

The ${ }^{13} \mathrm{C}$ NMR Spectrum of Compound 5 in DMSO- $d_{6}$ 

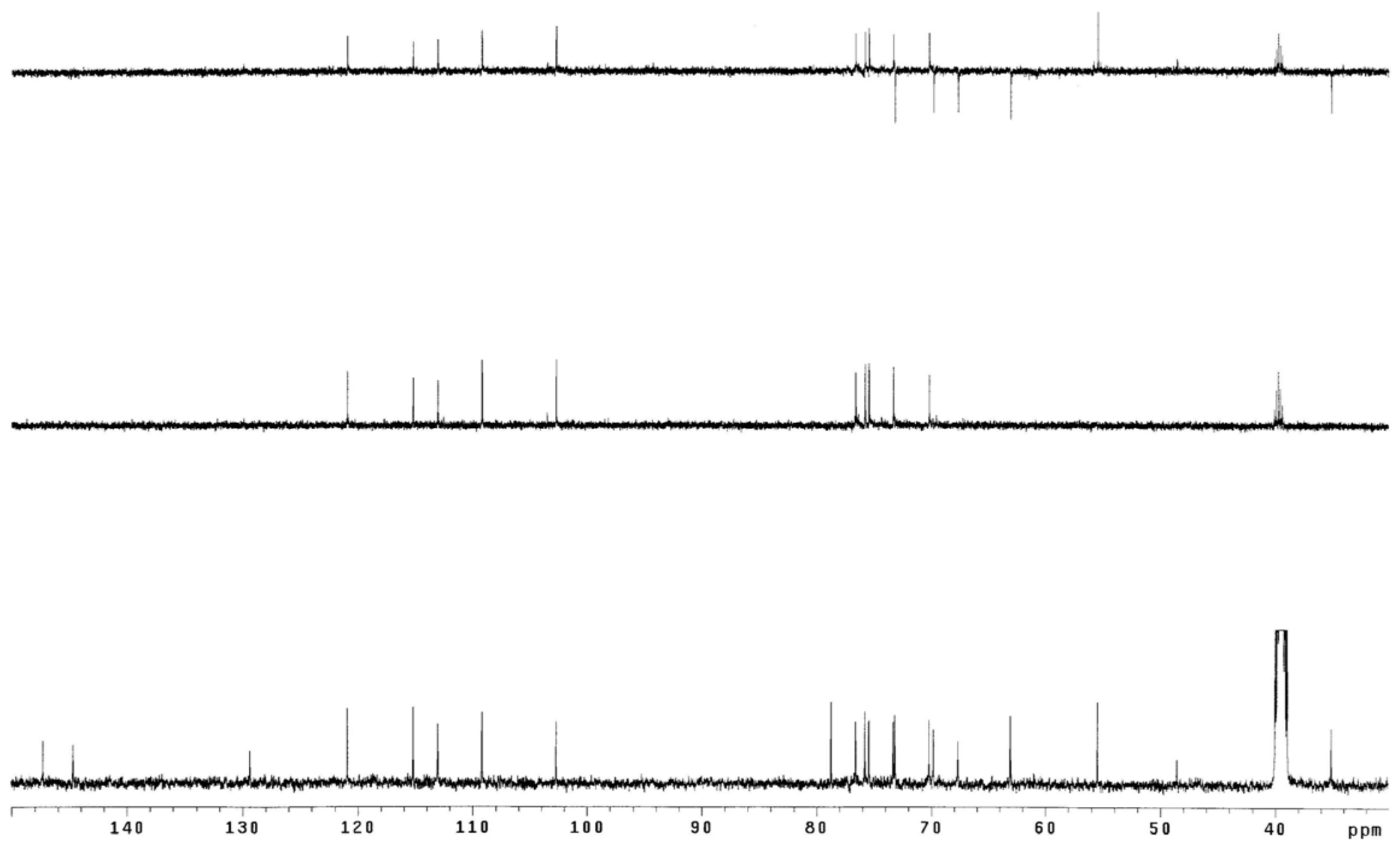

The DEPT Spectrum of Compound 5 in DMSO- $d_{6}$ 


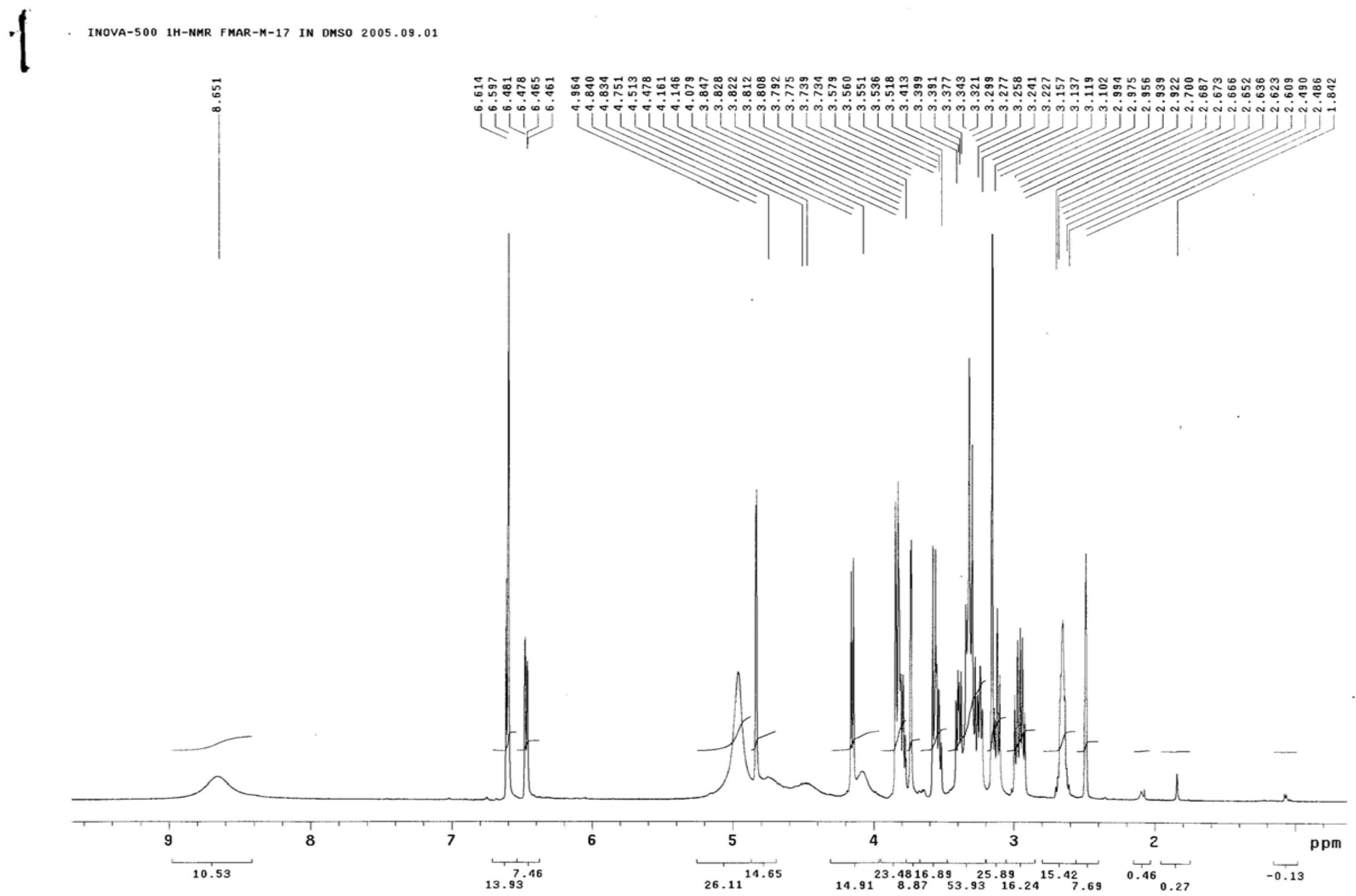

The ${ }^{1} \mathrm{H}$ NMR Spectrum of Compound 6 in DMSO- $d_{6}$ 
INOVA-500 13C-NMR FMAR-M-17 IN DMSO 2005.09.02
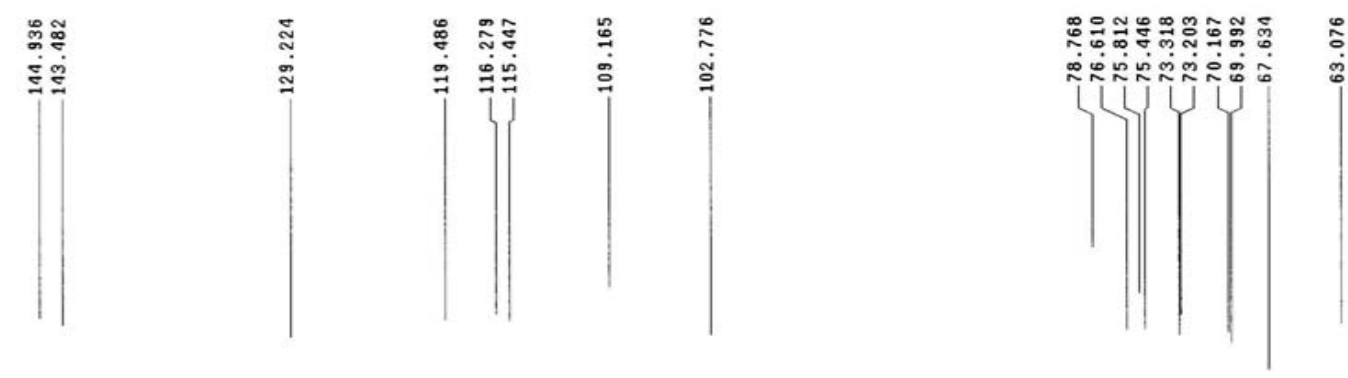

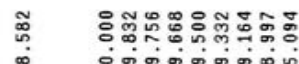

$$
\text { (1) }
$$

.

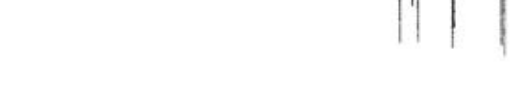




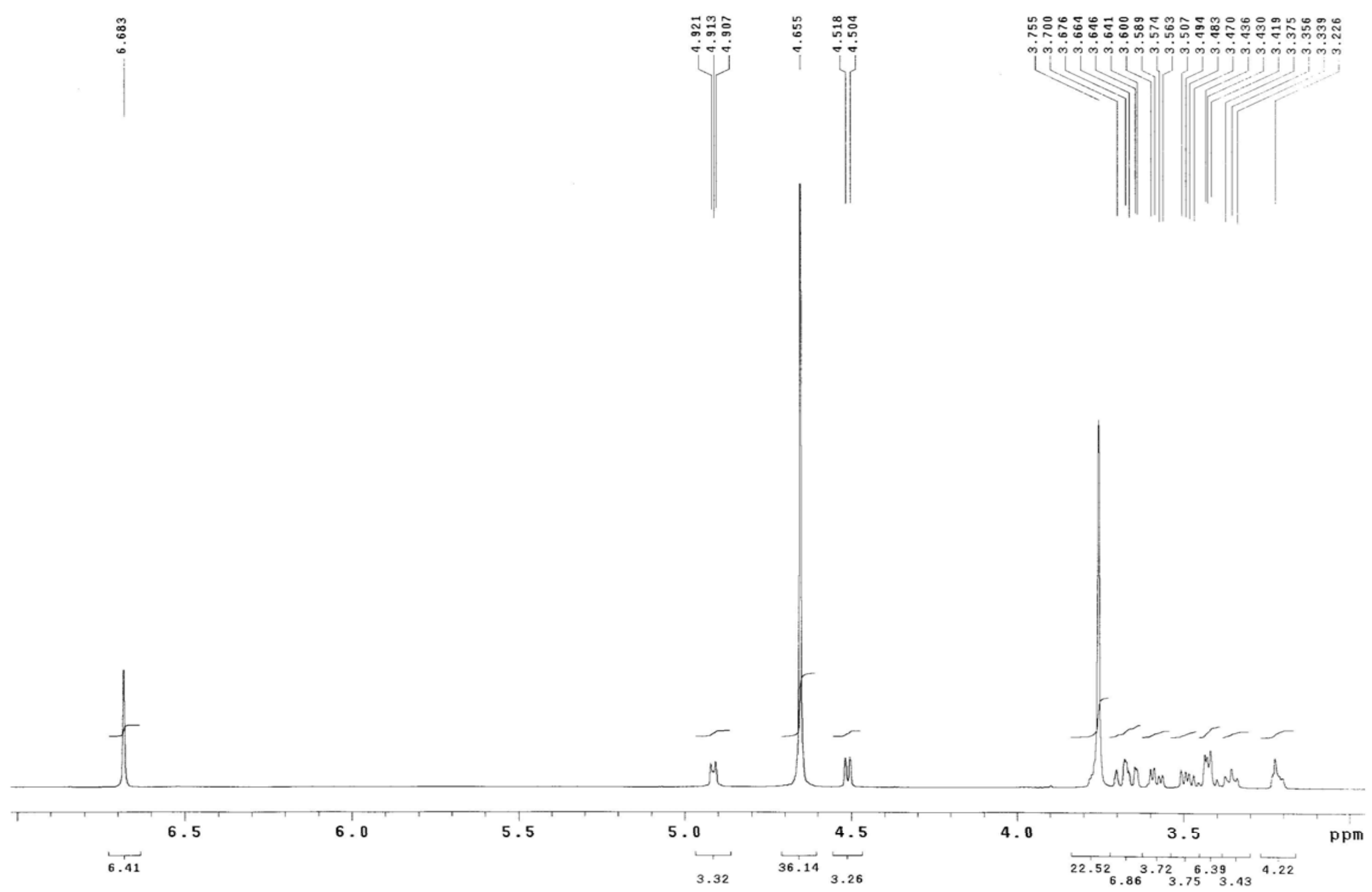

The ${ }^{1} \mathrm{H}$ NMR Spectrum of Compound 7 in $\mathrm{D}_{2} \mathrm{O}$ 
INOVA-500 13C-NMR FMAR-M-23A IN D20 2006.09.04
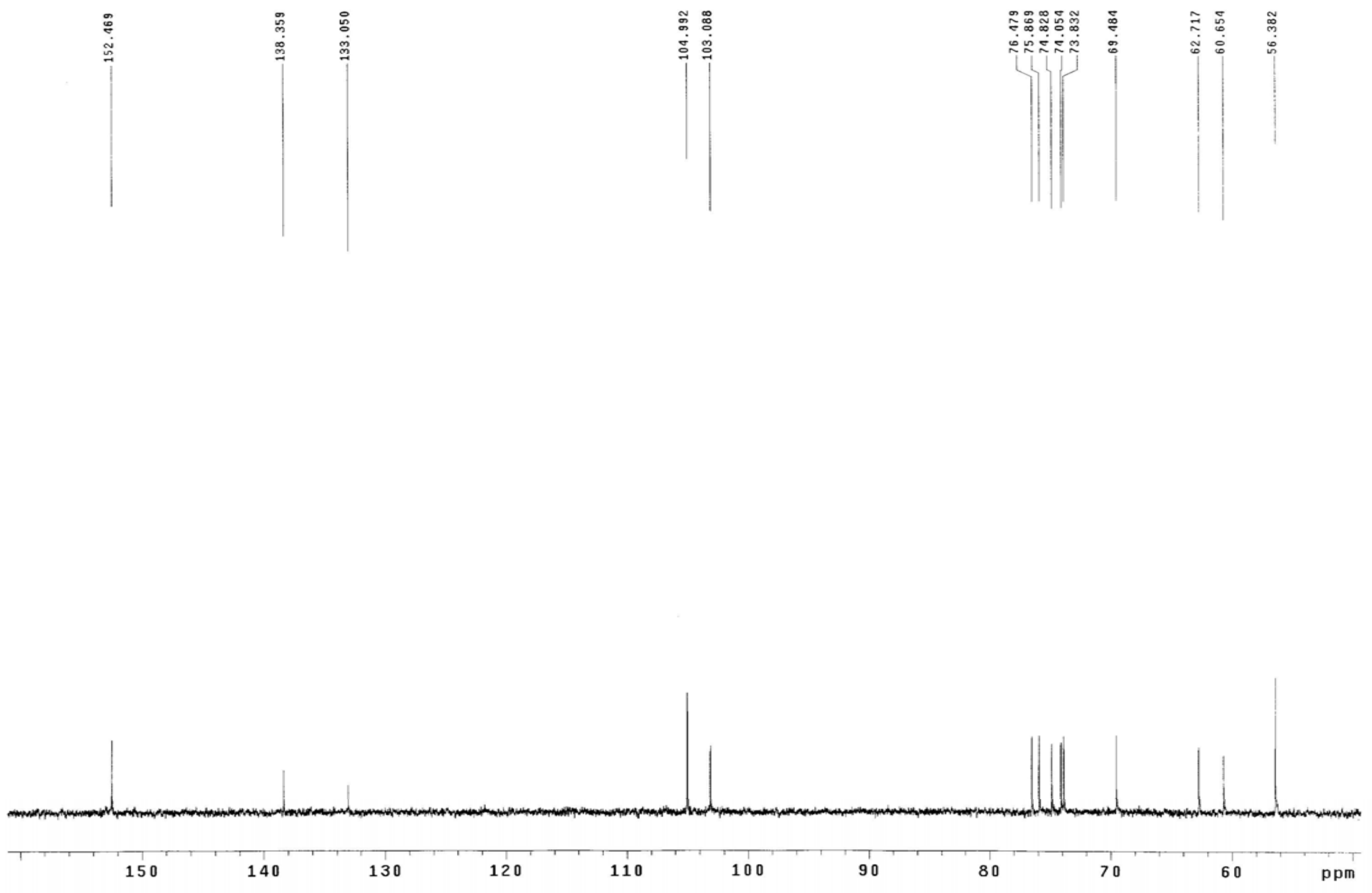

The ${ }^{13} \mathrm{C}$ NMR Spectrum of Compound 7 in $\mathrm{D}_{2} \mathrm{O}$ 

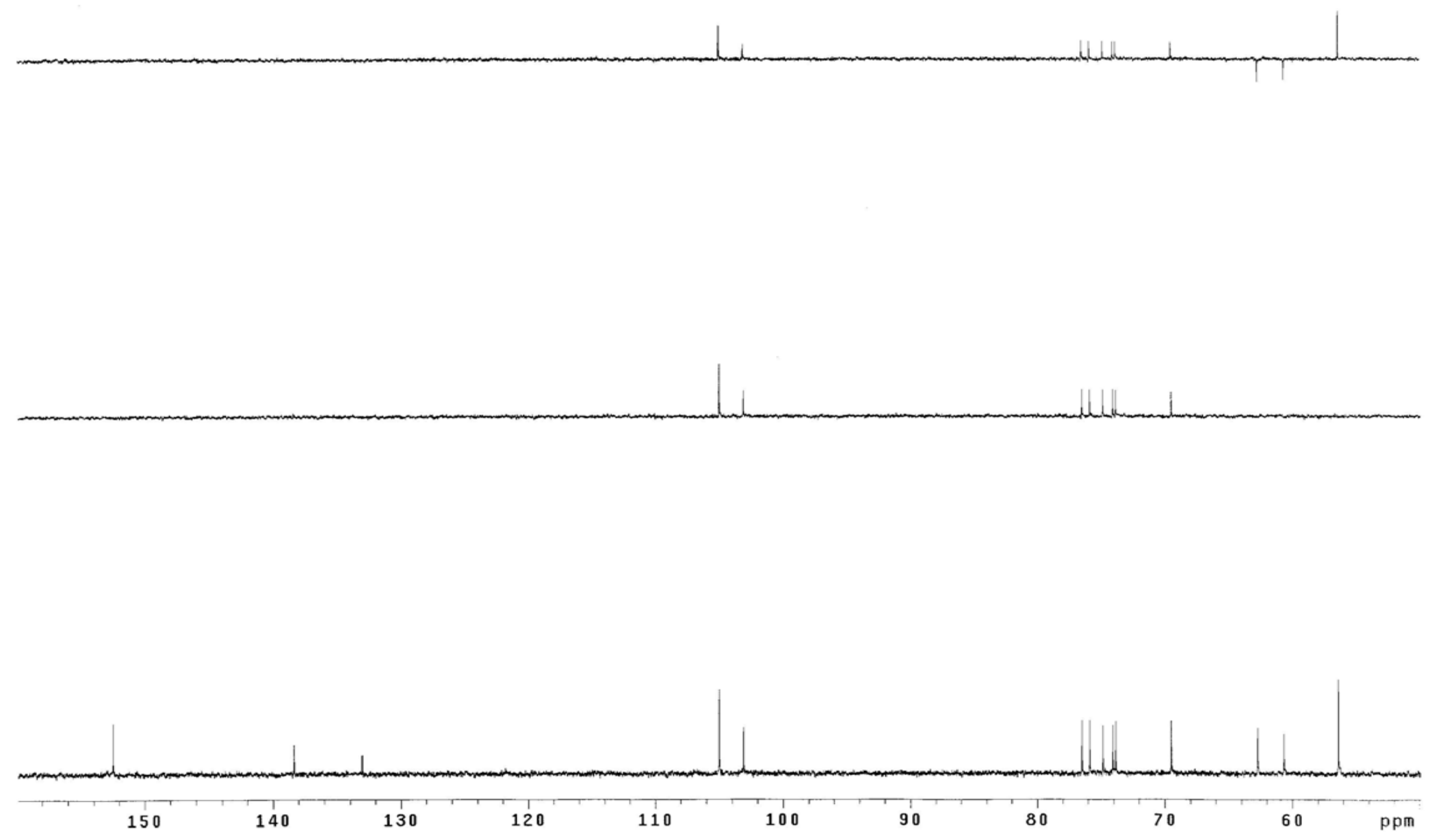

The DEPT Spectrum of Compound 7 in $\mathrm{D}_{2} \mathrm{O}$ 


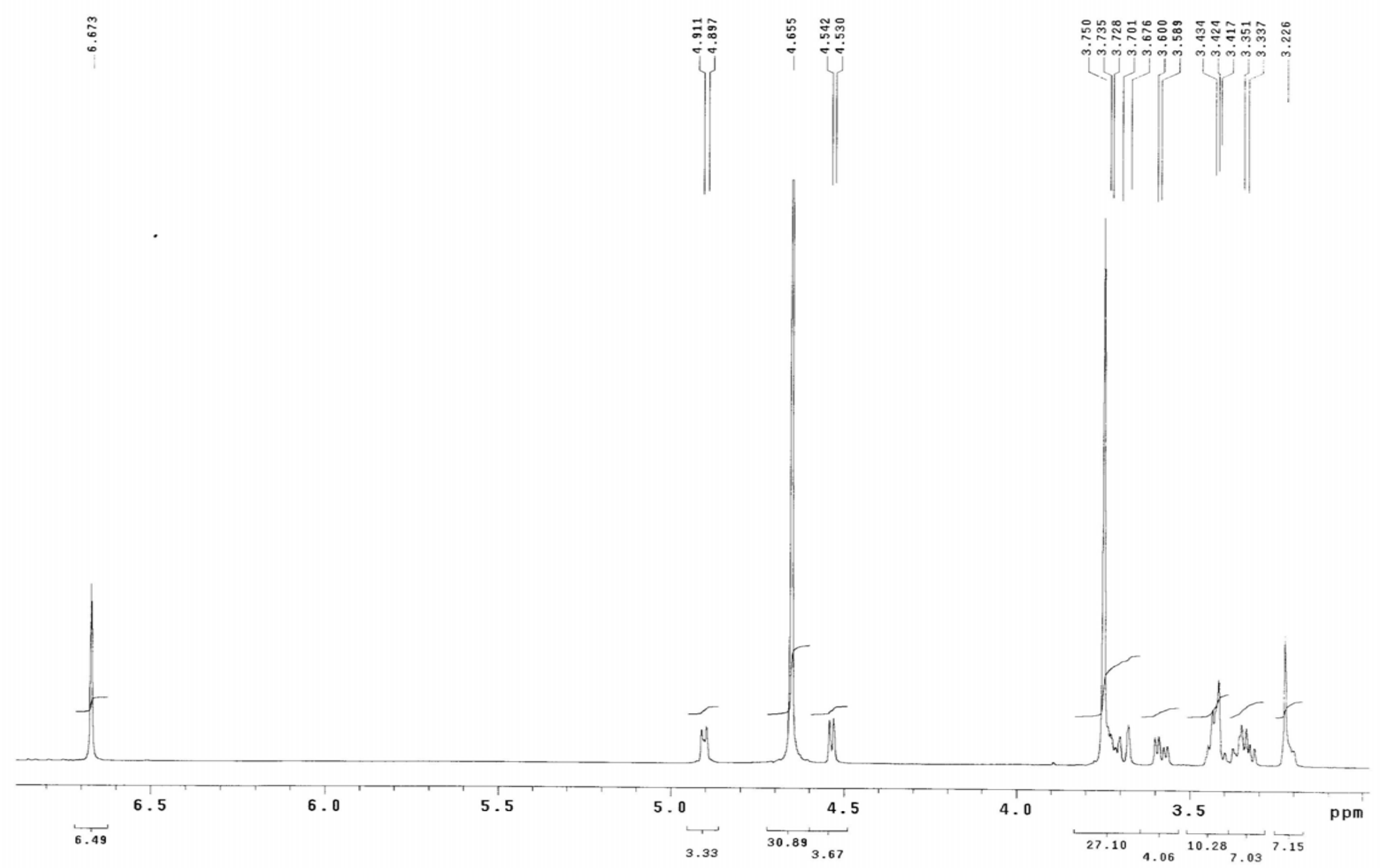

The ${ }^{1} \mathrm{H}$ NMR Spectrum of Compound 8 in $\mathrm{D}_{2} \mathrm{O}$ 
INOVA-500 13C-NMR FMAR-M-23C IN D20 2006.09.04
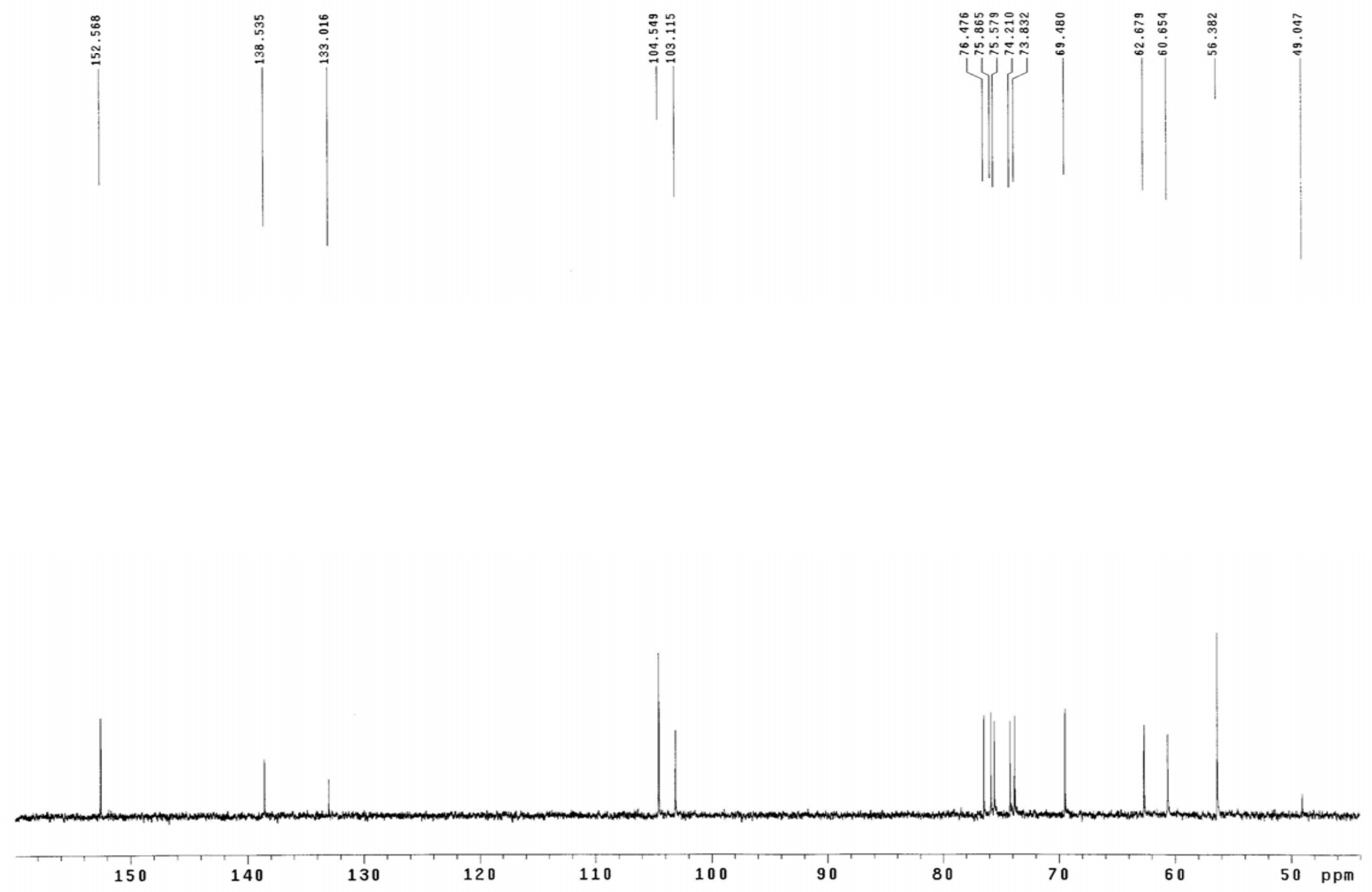

The ${ }^{13} \mathrm{C}$ NMR Spectrum of Compound 8 in $\mathrm{D}_{2} \mathrm{O}$ 

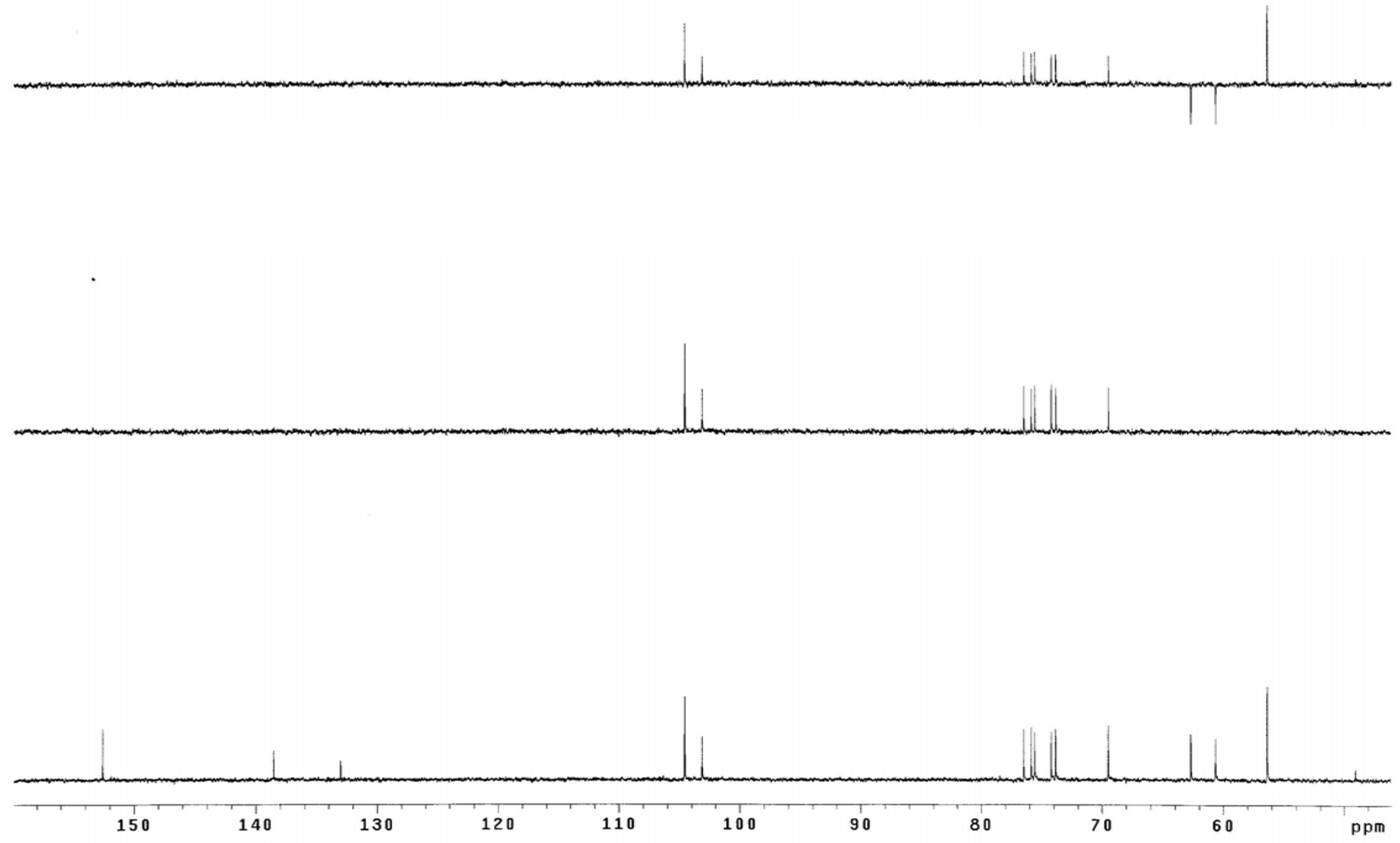

The DEPT Spectrum of Compound 8 in $\mathrm{D}_{2} \mathrm{O}$ 
MP-400 H1 D20 F . mar-m-23b

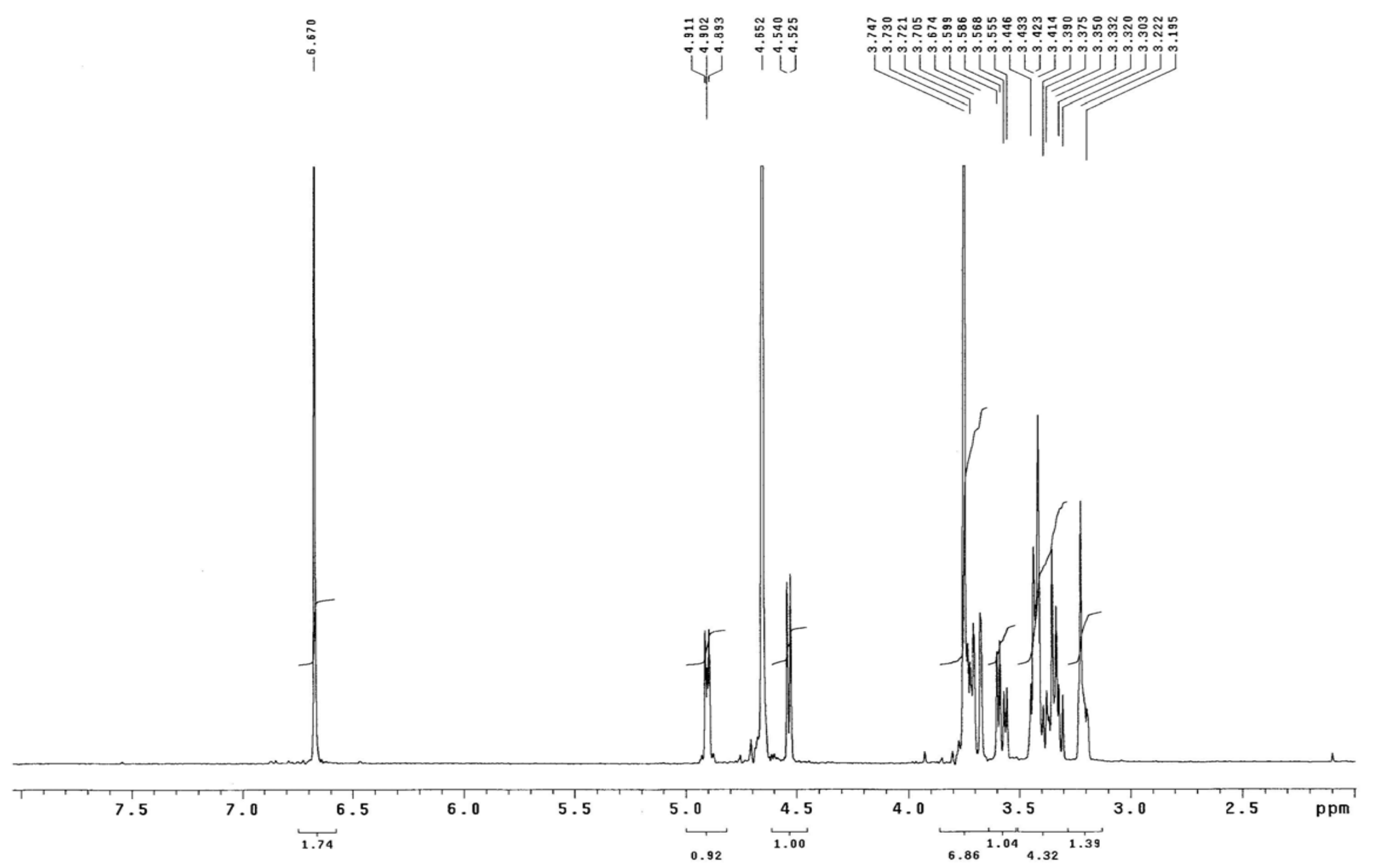

The ${ }^{1} \mathrm{H}$ NMR Spectrum of Compound 9 in $\mathrm{D}_{2} \mathrm{O}$ 
MP-400 C13 D20 F.mar-m-23b 060927
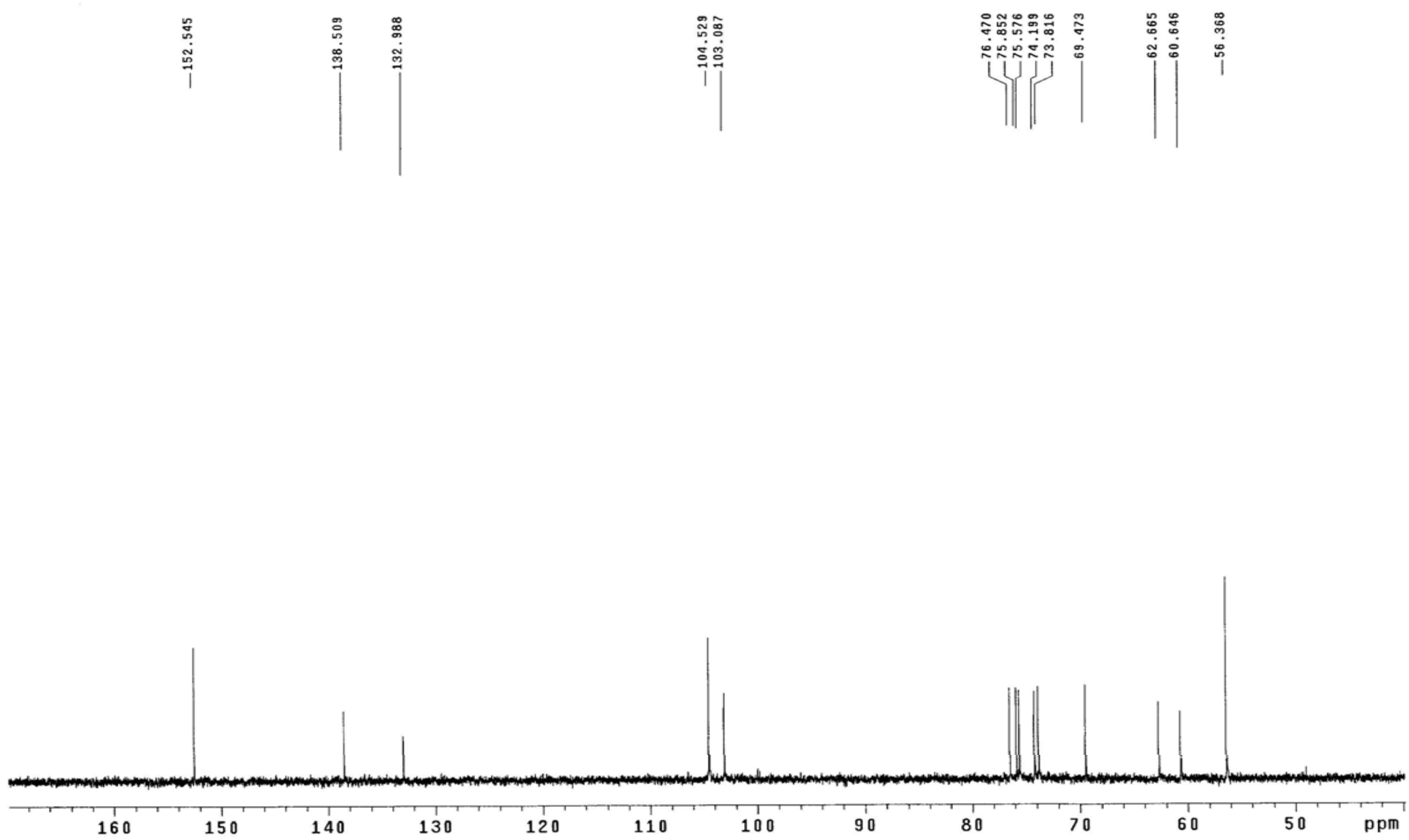

The ${ }^{13} \mathrm{C}$ NMR Spectrum of Compound 9 in $\mathrm{D}_{2} \mathrm{O}$ 
MP-400 DEPT D20 F.mar-m-23b 060927
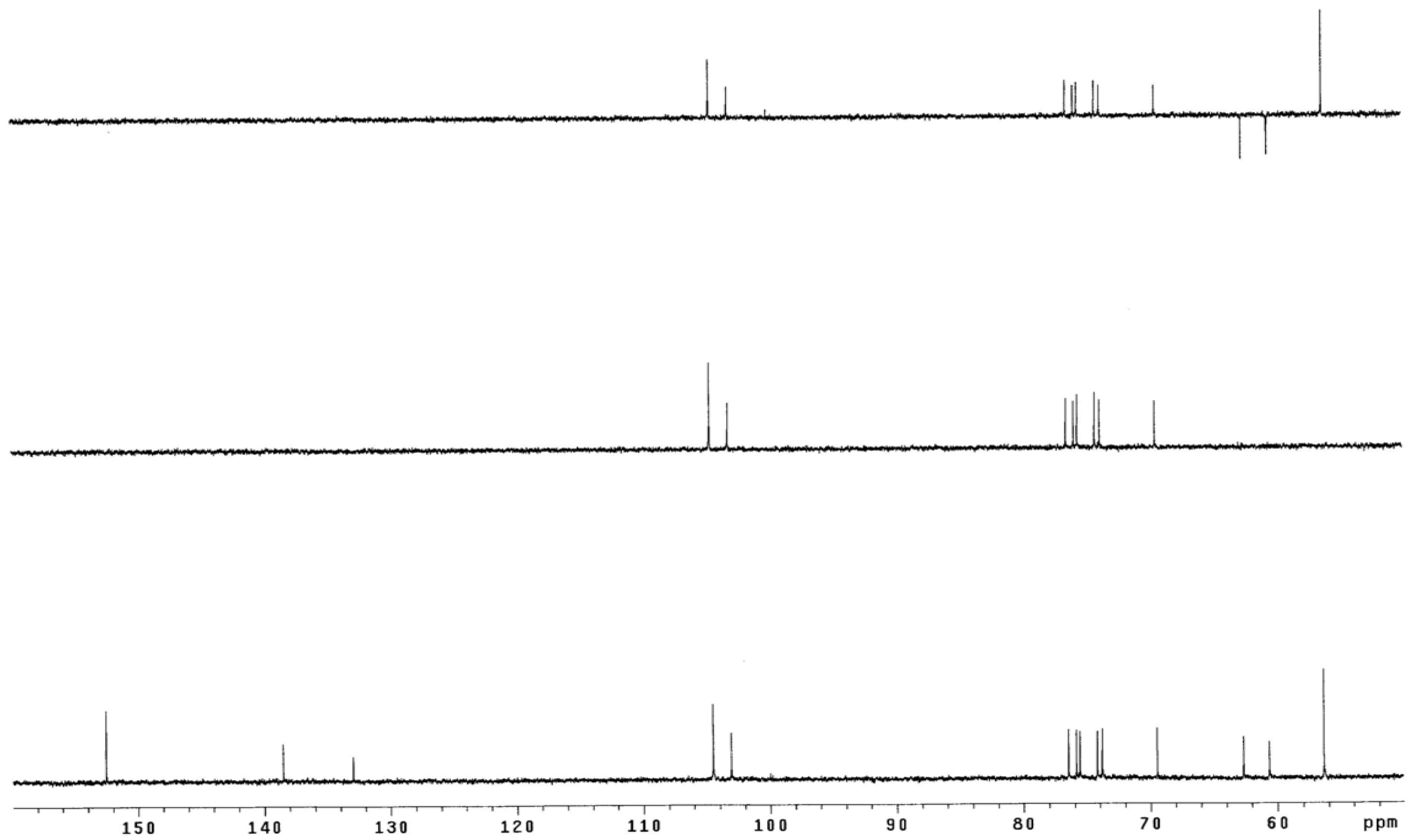

The DEPT Spectrum of Compound 9 in $D_{2} O$ 


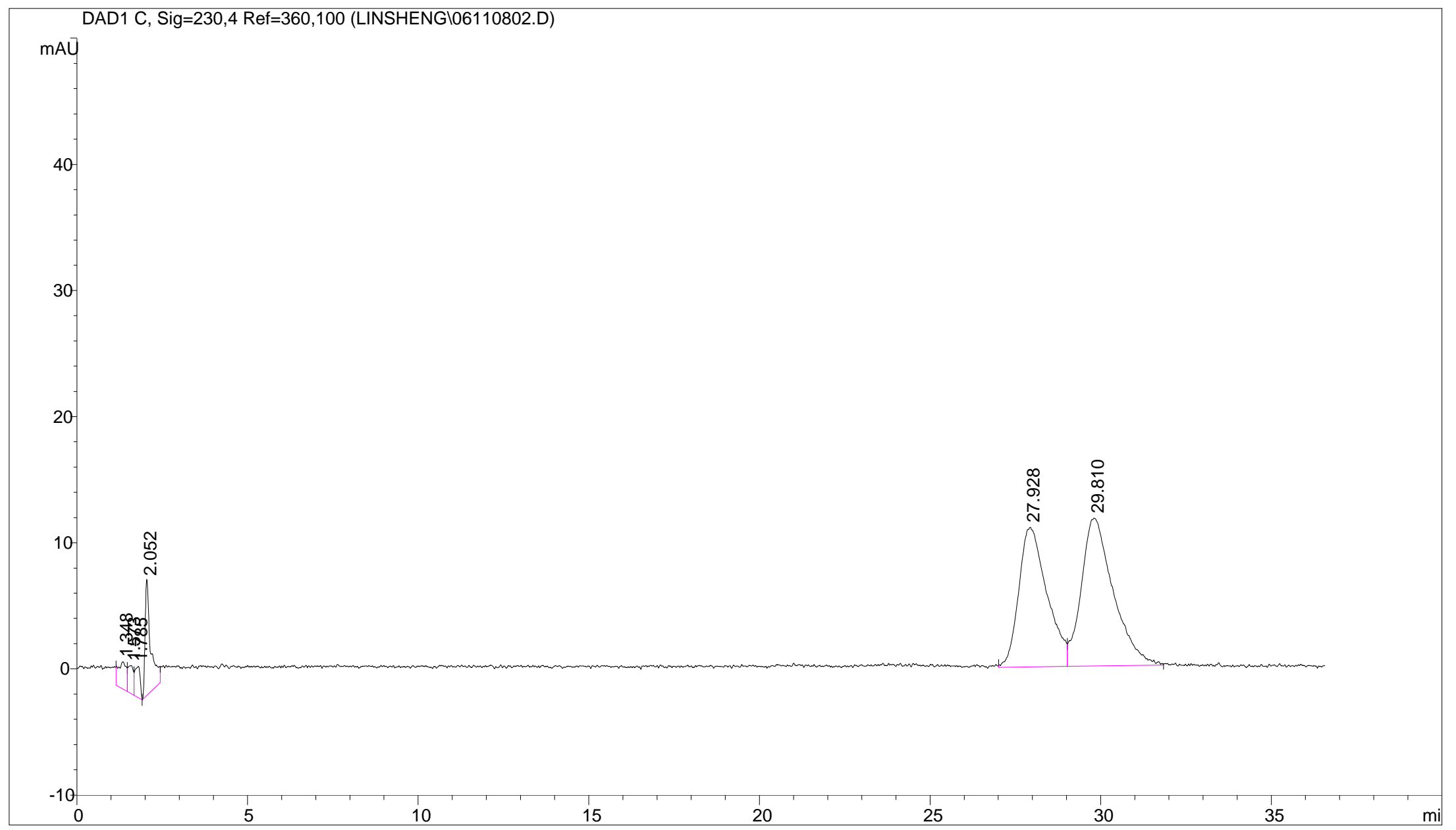

HPLC Instrument: Angilent 1100 system. Column: reversed phase C18, 250×4.6 mm, partical size $5 \mu$ (Brava, BDS, C18, $5 \mu$; Altech). Solvent: $0.6 \% \mathrm{MeCN}$ in $\mathrm{H}_{2} \mathrm{O}$. Flowrate: $1 \mathrm{~mL} / \mathrm{min}$.

The HPLC Spectrum of Compounds 8 and 9 
SI Table 1. ${ }^{13} \mathrm{C}$ NMR Data of 7-9 in Different Solvents (DMSO- $\left.d_{6}, \mathrm{C}_{5} \mathrm{D}_{5} \mathrm{~N}, \mathrm{D}_{2} \mathrm{O}\right)$

\begin{tabular}{|c|c|c|c|c|c|c|c|c|c|}
\hline \multirow[b]{3}{*}{ no. } & \multicolumn{3}{|c|}{ Erythro- arylglycerol } & \multicolumn{6}{|c|}{ Threo-arylglycerol } \\
\hline & \multicolumn{3}{|c|}{7} & \multicolumn{3}{|c|}{8} & \multicolumn{3}{|c|}{9} \\
\hline & DMSO- $d_{6}{ }^{a}$ & $\mathrm{C}_{5} \mathrm{D}_{5} \mathrm{~N}^{a}$ & $\mathrm{D}_{2} \mathrm{O}$ & DMSO- $d_{6}{ }^{a}$ & $\mathrm{C}_{5} \mathrm{D}_{5} \mathrm{~N}^{a}$ & $\mathrm{D}_{2} \mathrm{O}$ & DMSO- $d_{6}{ }^{a}$ & $\mathrm{C}_{5} \mathrm{D}_{5} \mathrm{~N}^{a}$ & $\mathrm{D}_{2} \mathrm{O}$ \\
\hline 1 & & 140.9 & 138.4 & 139.4 & 140.7 & 138.5 & 139.3 & 140.7 & 138.5 \\
\hline 2 & 105.5 & 106.2 & 105.0 & 104.9 & 105.7 & 104.5 & 104.9 & 105.8 & 104.5 \\
\hline 3 & 151.9 & 153.4 & 152.5 & 152.1 & 153.5 & 152.6 & 152.1 & 153.5 & 152.5 \\
\hline 4 & & 135.1 & 133.1 & 133.2 & 135.1 & 133.0 & 133.3 & 135.1 & 133.0 \\
\hline 5 & 151.9 & 153.4 & 152.5 & 152.1 & 153.5 & 152.6 & 152.1 & 153.5 & 152.5 \\
\hline 6 & 105.5 & 106.2 & 105.0 & 104.9 & 105.7 & 104.5 & 104.9 & 105.8 & 104.5 \\
\hline 7 & 73.5 & 76.2 & 74.1 & 72.4 & 74.7 & 74.2 & 72.5 & 74.7 & 74.2 \\
\hline 8 & 74.8 & 76.8 & 74.8 & 75.4 & 77.4 & 75.6 & 75.4 & 77.4 & 75.6 \\
\hline 9 & 63.0 & 64.9 & 62.7 & 62.5 & 64.4 & 62.7 & 62.5 & 64.4 & 62.7 \\
\hline $1^{\prime}$ & 102.9 & 105.4 & 103.1 & 102.8 & 105.3 & 103.1 & 102.8 & 105.3 & 103.1 \\
\hline $2^{\prime}$ & 74.2 & 76.1 & 73.8 & 74.0 & 76.1 & 73.8 & 74.0 & 76.2 & 73.8 \\
\hline $3^{\prime}$ & 76.7 & 78.7 & 75.9 & 77.1 & 78.7 & 75.9 & 77.1 & 78.7 & 75.9 \\
\hline $4^{\prime}$ & 69.4 & 71.6 & 69.5 & 69.8 & 71.6 & 69.5 & 69.8 & 71.6 & 69.5 \\
\hline $5^{\prime}$ & 76.5 & 78.4 & 76.5 & 76.4 & 78.4 & 76.5 & 76.3 & 78.4 & 76.5 \\
\hline $6^{\prime}$ & 60.5 & 62.6 & 60.6 & 60.9 & 62.6 & 60.7 & 60.8 & 62.6 & 60.6 \\
\hline OMe & 56.3 & 56.4 & 56.4 & 56.3 & 56.4 & 56.4 & 56.3 & 56.4 & 56.4 \\
\hline$\Delta \delta_{\mathrm{C} 8-\mathrm{C} 7}^{b}$ & 1.3 & 0.6 & 0.7 & 3.0 & 2.7 & 1.4 & 2.9 & 2.7 & 1.4 \\
\hline
\end{tabular}

${ }^{a}{ }^{13} \mathrm{C}$ NMR data were measured for 7-9 at 125 or $100 \mathrm{MHz}$. The assignments were based on a comparison with those of 7-9 in $\mathrm{D}_{2} \mathrm{O}$ and analogs reported in literatures. 
SI Table 2. ${ }^{13} \mathrm{C}$ NMR Data of 7a-9a in Different Solvents (DMSO- $d_{6}, \mathrm{C}_{5} \mathrm{D}_{5} \mathrm{~N}, \mathrm{MeOH}-d_{4}$, and $\left.\mathrm{Me}_{2} \mathrm{CO}-d_{6}\right)^{a}$

\begin{tabular}{|c|c|c|c|c|c|c|c|c|c|c|c|c|}
\hline \multirow[b]{3}{*}{ No. } & \multicolumn{4}{|c|}{ Erythro-arylglycerol } & \multicolumn{8}{|c|}{ Threo-arylglycerol } \\
\hline & \multicolumn{4}{|c|}{7 a } & \multicolumn{4}{|c|}{$8 a$} & \multicolumn{4}{|c|}{ 9a } \\
\hline & DMSO- $d_{6}$ & $\mathrm{C}_{5} \mathrm{D}_{5} \mathrm{~N}$ & $\mathrm{MeOH}-d_{4}$ & $\mathrm{Me}_{2} \mathrm{CO}-d_{6}$ & DMSO- $d_{6}$ & $\mathrm{C}_{5} \mathrm{D}_{5} \mathrm{~N}$ & $\mathrm{MeOH}-d_{4}$ & $\mathrm{Me}_{2} \mathrm{CO}-d_{6}$ & DMSO- $d_{6}$ & $\mathrm{C}_{5} \mathrm{D}_{5} \mathrm{~N}$ & $\mathrm{MeOH}-d_{4}$ & $\mathrm{Me}_{2} \mathrm{CO}-d_{6}$ \\
\hline $\begin{array}{l}1 \\
2\end{array}$ & $\begin{array}{l}134.1 \\
104.5\end{array}$ & $\begin{array}{l}136.7 \\
105.8\end{array}$ & $\begin{array}{l}136.0 \\
105.5\end{array}$ & $\begin{array}{l}136.0 \\
105.3\end{array}$ & $\begin{array}{l}134.1 \\
104.0\end{array}$ & $\begin{array}{l}135.8 \\
105.5\end{array}$ & $\begin{array}{l}136.0 \\
105.2\end{array}$ & $\begin{array}{l}136.0 \\
105.1\end{array}$ & $\begin{array}{l}134.1 \\
104.0\end{array}$ & $\begin{array}{l}135.7 \\
105.5\end{array}$ & $\begin{array}{l}136.0 \\
105.2\end{array}$ & $\begin{array}{l}136.0 \\
105.1\end{array}$ \\
\hline 3 & 147.3 & 148.8 & 149.0 & 148.3 & 147.4 & 148.9 & 149.1 & 148.4 & 147.4 & 149.0 & 149.1 & 148.4 \\
\hline 4 & 133.5 & 134.7 & 134.1 & 134.0 & 133.4 & 134.5 & 134.1 & 133.9 & 133.4 & 134.5 & 134.1 & 133.9 \\
\hline 5 & 147.3 & 148.8 & 149.0 & 148.3 & 147.4 & 148.9 & 149.1 & 148.4 & 147.4 & 149.0 & 149.1 & 148.4 \\
\hline 6 & 104.5 & 105.8 & 105.5 & 105.3 & 104.0 & 105.5 & 105.2 & 105.1 & 104.0 & 105.5 & 105.2 & 105.1 \\
\hline 7 & 74.0 & 76.5 & 76.3 & 76.2 & 72.9 & 75.1 & 75.6 & 75.0 & 72.9 & 75.1 & 75.6 & 75.0 \\
\hline 8 & 75.3 & 77.0 & 76.7 & 76.4 & 75.8 & 77.9 & 77.6 & 77.2 & 75.8 & 77.8 & 77.6 & 77.3 \\
\hline 9 & 62.9 & 65.0 & 64.5 & 64.4 & 62.5 & 64.4 & 64.3 & 64.0 & 62.5 & 64.4 & 64.2 & 64.0 \\
\hline OMe & 55.9 & 56.2 & 56.7 & 56.5 & 55.8 & 56.2 & 56.7 & 56.6 & 55.8 & 56.3 & 56.7 & 56.6 \\
\hline$\Delta \delta_{\mathrm{CB}-\mathrm{C} 7}$ & 1.3 & 0.5 & 0.4 & 0.2 & 2.9 & 2.8 & 2.0 & 2.2 & 2.9 & 2.7 & 2.0 & 2.3 \\
\hline
\end{tabular}

${ }^{a}{ }^{13} \mathrm{C}$ NMR data were measured for $\mathbf{7 a - 9 a}$ at 125 or $100 \mathrm{MHz}$. The assignments were based on a comparison with those of 7-9 in $\mathrm{D}_{2} \mathrm{O}$ and analogs reported in literatures. 
SI Table 3. $\quad{ }^{1} \mathrm{H}$ NMR Data of 7-9 in DMSO- $d_{6}$ and $\mathrm{C}_{5} \mathrm{D}_{5} \mathrm{~N}^{a}$

\begin{tabular}{|c|c|c|c|c|c|c|}
\hline \multirow[b]{2}{*}{ no. } & \multicolumn{2}{|c|}{7} & \multicolumn{2}{|c|}{8} & \multicolumn{2}{|c|}{9} \\
\hline & DMSO- $d_{6}$ & Pyridine- $d_{5}$ & DMSO- $d_{6}$ & Pyridine- $d_{5}$ & DMSO- $d_{6}$ & Pyridine- $d_{5}$ \\
\hline 2 & $6.64 \mathrm{~s}$ & $7.19 \mathrm{~s}$ & $6.64 \mathrm{~s}$ & $7.19 \mathrm{~s}$ & $6.64 \mathrm{~s}$ & $7.19 \mathrm{~s}$ \\
\hline 6 & $6.64 \mathrm{~s}$ & $7.19 \mathrm{~s}$ & $6.64 \mathrm{~s}$ & $7.19 \mathrm{~s}$ & $6.64 \mathrm{~s}$ & $7.19 \mathrm{~s}$ \\
\hline 7 & 4.34 dd $(4.5,5.0)$ & $5.28 \mathrm{~d}(5.6)$ & $4.46 \mathrm{~d}(5.5)$ & $5.35 \mathrm{~d}(4.4)$ & $4.46 \mathrm{~d}(5.5)$ & $5.35 \mathrm{~d}(4.4)$ \\
\hline 8 & $3.48 \mathrm{~m}$ & $4.44 \mathrm{~m}$ & $3.47 \mathrm{~m}$ & $4.36 \mathrm{~m}$ & $3.48 \mathrm{~m}$ & $4.36 \mathrm{~m}$ \\
\hline 9a & 3.46 dd $(11.5,4.0)$ & 4.44 dd $(11.2,4.0)$ & 3.35 dd $(11.5,4.0)$ & 4.26 dd $(11.2,4.0)$ & 3.35 dd $(11.5,4.0)$ & $4.26 \mathrm{dd}(11.2,4.0)$ \\
\hline $9 b$ & 3.38 dd $(11.5,6.5)$ & 4.43 dd $(11.2,6.4)$ & 3.17dd (11.5,6.5) & 4.13 dd $(11.2,6.4)$ & 3.17 dd $(11.5,6.5)$ & $4.12 \mathrm{~d}(11.2,6.4)$ \\
\hline $1^{\prime}$ & $4.84 \mathrm{~d}(8.0)$ & $5.71 \mathrm{~d}(6.0)$ & $4.84 \mathrm{~d}(8.0)$ & $5.71 \mathrm{~d}(6.4)$ & $4.85 \mathrm{~d}(7.0)$ & $5.73 \mathrm{~d}(6.0)$ \\
\hline $2^{\prime}$ & 3.19 ddd $(8.5,8.0,5.0)$ & $4.32 \mathrm{dd}(7.5,6.0)$ & 3.20 ddd $(8.5,8.0,5.0)$ & $4.32 \mathrm{dd}(7.5,6.0)$ & 3.19 ddd $(8.5,7.0,5.0)$ & $4.25 \mathrm{dd} \quad(7.5,6.0)$ \\
\hline $3^{\prime}$ & 3.17dt $(8.5,5.0)$ & 4.38 dd $(6.5,6.0)$ & $3.19 \mathrm{dt}(8.5,5.0)$ & 4.35 dd $(6.5,6.0))$ & $3.19 \mathrm{dt}(8.5,5.0)$ & 4.12 dd $(6.5,6.0)$ \\
\hline $4^{\prime}$ & 3.02ddd(9.0,8.5,5.0) & 4.31dd $(6.5,6.0)$ & 3.02 ddd $(9.0,8.5,5.0)$ & 4.30 dd $(6.5,6.0)$ & 3.02 ddd $(9.0,8.5,5.0)$ & 4.31 dd $(6.5,6.0)$ \\
\hline $5^{\prime}$ & $3.18 \mathrm{~m}$ & $3.92 \mathrm{~m}$ & $3.18 \mathrm{~m}$ & $3.92 \mathrm{~m}$ & $3.18 \mathrm{~m}$ & $3.92 \mathrm{~m}$ \\
\hline $6^{\prime} \mathrm{a}$ & 3.58ddd (11.5,5.5,2.0) & 4.39dd $(10.4,1.6)$ & 3.58 ddd $(11.5,5.5,2.0)$ & $4.38 \mathrm{dd}(10.4,1.6)$ & 3.58 ddd $(11.5,5.5,2.0)$ & 4.38 dd $(10.4,1.6)$ \\
\hline $6^{\prime} b$ & 3.40ddd (11.5,6.0,5.0) & 4.25dd $(10.4,4.0)$ & 3.40 ddd $(11.5,6.0,5.0)$ & 4.25 dd $(10.4,4.0)$ & 3.40 ddd $(11.5,6.0,5.0)$ & 4.25 dd $(10.4,4.0)$ \\
\hline $\mathrm{OCH}_{3}$ & $3.73 \mathrm{~s}$ & $3.66 \mathrm{~s}$ & $3.73 \mathrm{~s}$ & $3.67 \mathrm{~s}$ & $3.73 \mathrm{~s}$ & $3.67 \mathrm{~s}$ \\
\hline
\end{tabular}

${ }^{a}{ }^{1} \mathrm{H}$ NMR data were measured for 7-9 at 500 or $400 \mathrm{MHz}$. Proton coupling constants $(\mathrm{J})$ in $\mathrm{Hz}$ are given in parentheses. The assignments were based on a comparison with those of 7-9 in $\mathrm{D}_{2} \mathrm{O}$ and analogs reported in literatures. 
SI Table 4. ${ }^{1} \mathrm{H}$ NMR Data of 7a-9a in $\mathrm{Me}_{2} \mathrm{CO}-d_{6}$, MeOH- $d_{4}$, DMSO- $d_{6}$, and $\mathrm{C}_{5} \mathrm{D}_{5} \mathrm{~N}^{a}$

\begin{tabular}{|c|c|c|c|c|c|c|c|c|c|c|c|c|}
\hline \multirow[b]{2}{*}{ no. } & \multicolumn{4}{|c|}{$7 a$} & \multicolumn{4}{|c|}{$8 a$} & \multicolumn{4}{|c|}{$9 a$} \\
\hline & $\mathrm{Me}_{2} \mathrm{CO}-\mathrm{d}_{6}$ & $\mathrm{MeOH}-d_{4}$ & DMSO- $d_{6}$ & Pyridine- $d_{5}$ & $\mathrm{Me}_{2} \mathrm{CO}-d_{6}$ & $\mathrm{MeOH}-d_{4}$ & DMSO- $d_{6}$ & Pyridine- $d_{5}$ & $\mathrm{Me}_{2} \mathrm{CO}-d_{6}$ & $\mathrm{MeOH}-d_{4}$ & DMSO- $d_{6}$ & Pyridine- $d_{5}$ \\
\hline 2 & $6.69 \mathrm{~s}$ & $6.64 \mathrm{~s}$ & $6.57 \mathrm{~s}$ & $7.21 \mathrm{~s}$ & $6.69 \mathrm{~s}$ & $6.63 \mathrm{~s}$ & $6.57 \mathrm{~s}$ & $7.19 \mathrm{~s}$ & $6.69 \mathrm{~s}$ & $6.63 \mathrm{~s}$ & $6.57 \mathrm{~s}$ & $7.19 \mathrm{~s}$ \\
\hline 6 & $6.69 \mathrm{~s}$ & $6.64 \mathrm{~s}$ & $6.57 \mathrm{~s}$ & $7.21 \mathrm{~s}$ & $6.69 \mathrm{~s}$ & $6.63 \mathrm{~s}$ & $6.57 \mathrm{~s}$ & $7.19 \mathrm{~s}$ & $6.69 \mathrm{~s}$ & $6.63 \mathrm{~s}$ & $6.57 \mathrm{~s}$ & $7.19 \mathrm{~s}$ \\
\hline 7 & $\begin{array}{l}\text { 4.55dd } \\
(4.8,5.6)\end{array}$ & $\begin{array}{l}4.46 \mathrm{~d} \\
(6.0)\end{array}$ & 4.32 brd s & $\begin{array}{l}5.32 \mathrm{~d} \\
(5.0)\end{array}$ & $\begin{array}{l}\text { 44.55dd } \\
(5.2,5.2)\end{array}$ & $\begin{array}{l}4.47 \mathrm{~d} \\
(6.0)\end{array}$ & $\begin{array}{l}4.38 \mathrm{dd} \\
(5.0,6.0)\end{array}$ & $\begin{array}{l}5.33 \mathrm{~d} \\
(5.6)\end{array}$ & $\begin{array}{l}\text { 4.55dd } \\
(4.0,5.6)\end{array}$ & $\begin{array}{l}4.47 \mathrm{~d} \\
(6.4)\end{array}$ & $\begin{array}{l}4.38 \mathrm{dd} \\
(5.0,6.0)\end{array}$ & $\begin{array}{l}5.33 \mathrm{~d} \\
(5.6)\end{array}$ \\
\hline 8 & $3.62 \mathrm{~m}$ & 3.67 m & $3.48 \mathrm{~m}$ & $4.51 \mathrm{~m}$ & $3.62 \mathrm{~m}$ & $3.61 \mathrm{~m}$ & $3.44 \mathrm{~m}$ & $4.43 \mathrm{~m}$ & $3.61 \mathrm{~m}$ & $3.61 \mathrm{~m}$ & $3.44 \mathrm{~m}$ & $4.43 \mathrm{~m}$ \\
\hline $9 a$ & $\begin{array}{l}\text { 3.49dd } \\
(11.2,4.0)\end{array}$ & $\begin{array}{l}3.60 \mathrm{dd} \\
(11.5,4.0)\end{array}$ & $\begin{array}{l}3.46 \mathrm{dd} \\
(11.5,4.0)\end{array}$ & $\begin{array}{l}4.47 \mathrm{dd} \\
(11.2,4.0)\end{array}$ & $\begin{array}{l}3.49 \mathrm{dd} \\
(11.2,4.0)\end{array}$ & $\begin{array}{l}3.44 \mathrm{dd} \\
(11.2,4.0)\end{array}$ & $\begin{array}{l}3.31 \mathrm{dd} \\
(11.5,4.0)\end{array}$ & $\begin{array}{l}4.27 \mathrm{dd} \\
(11.2,4.0)\end{array}$ & $\begin{array}{l}3.49 \mathrm{dd} \\
(11.2,4.0)\end{array}$ & $\begin{array}{l}3.44 \mathrm{dd} \\
(11.2,4.0)\end{array}$ & $\begin{array}{l}3.32 \text { dd } \\
(11.5,4.0)\end{array}$ & $\begin{array}{l}4.27 \mathrm{dd} \\
(11.2,4.0)\end{array}$ \\
\hline $9 b$ & $\begin{array}{l}3.41 \mathrm{dd} \\
(11.2,6.4)\end{array}$ & $\begin{array}{l}3.53 \mathrm{dd} \\
(11.5,6.5)\end{array}$ & $\begin{array}{l}3.36 \mathrm{dd} \\
(11.5,6.5)\end{array}$ & $\begin{array}{l}4.45 \mathrm{dd} \\
(11.5,6.5)\end{array}$ & $\begin{array}{l}3.40 \mathrm{dd} \\
(11.2,6.4)\end{array}$ & $\begin{array}{l}3.31 \mathrm{dd} \\
(11.2,6.0)\end{array}$ & $\begin{array}{l}3.15 \mathrm{dd} \\
(11.5,6.5)\end{array}$ & $\begin{array}{l}4.13 \mathrm{dd} \\
(11.2,6.4)\end{array}$ & $\begin{array}{l}3.41 \mathrm{dd} \\
(11.2,6.4)\end{array}$ & $\begin{array}{l}3.31 \mathrm{dd} \\
(11.2,6.0)\end{array}$ & $\begin{array}{l}3.15 \mathrm{dd} \\
(11.5,6.5)\end{array}$ & $\begin{array}{l}4.13 \mathrm{dd} \\
(11.2,6.4)\end{array}$ \\
\hline $\mathrm{OCH}_{3}$ & $3.80 \mathrm{~s}$ & $3.79 \mathrm{~s}$ & $3.72 \mathrm{~s}$ & $3.71 \mathrm{~s}$ & $3.80 \mathrm{~s}$ & $3.79 \mathrm{~s}$ & $3.72 \mathrm{~s}$ & $3.72 \mathrm{~s}$ & $3.80 \mathrm{~s}$ & $3.79 \mathrm{~s}$ & $3.72 \mathrm{~s}$ & $3.72 \mathrm{~s}$ \\
\hline
\end{tabular}

${ }^{a}{ }_{1} \mathrm{H}$ NMR data were measured for 7a-9a at 500 or $400 \mathrm{MHz}$. Proton coupling constants $(J)$ in $\mathrm{Hz}$ are given in parentheses. 